\title{
Numerical and Experimental Investigations on Seismic Response of Building Frames under Influence of Soil-Structure Interaction
}

\author{
S. Hamid Reza Tabatabaiefar*, Behzad Fatahi and Bijan Samali \\ Centre for Built Infrastructure Research, School of Civil and Environmental Engineering, University of Technology Sydney \\ (UTS), Australia
}

(Received: 28 February 2013; Received revised form: 31 October 2013; Accepted: 28 November 2013)

\begin{abstract}
In this study, an enhanced numerical soil-structure model has been developed which treats the behaviour of soil and structure with equal rigour. The proposed numerical soil-structure model has been verified and validated by performing experimental shaking table tests. To achieve this goal, a series of experimental shaking table tests were performed on the physical fixed based (structure directly fixed on top of the shaking table) and flexible base (considering soil and structure) models under the influence of four scaled earthquake acceleration records and the results were measured. Comparing the experimental results with the numerical analysis predictions, it is noted that the numerical predictions and laboratory measurements are in a good agreement. Thus, the proposed numerical soil-structure model is a valid and qualified method of simulation with sufficient accuracy which can be employed for further numerical soil-structure interaction investigation studies. Based on the predicted and observed values of lateral deflections of fixed base and flexible base models, lateral deflections of the flexible base model have noticeably amplified in comparison to the fixed base model. As a result of the lateral deflection amplifications, it is observed that the performance level of the scaled structural model changed significantly which could be safety threatening.
\end{abstract}

Key words: soil-structure interaction, seismic response, shaking table test, performance level.

\section{INTRODUCTION}

Soil-Structure Interaction (SSI) includes a set of mechanisms accounting for the flexibility of the foundation support beneath a given structure resulting in altering the ground motion in the vicinity of the foundation compared to the free-field. It determines the actual loading experienced by the soil-structure system resulting from the free-field seismic ground motions. Wolf (1985) elucidated that the seismic excitation experienced by structures is a function of the earthquake characteristics, travel path effects, local site effects, and soil-structure interaction effects. The result of the first three of these factors can be summarised as free-field ground motion. Krawinkler et al. (2003) elucidated that structural response to the free-field motion is influenced by SSI. In particular, accelerations within the structure are affected by the flexibility of the foundation and the difference between foundation and free-field motions (Tabatabaiefar et al. 2013; Turan et al. 2013).

The importance of soil-structure interaction both for static and dynamic loads has been well established and the related literature covers at least 30 years of computational and analytical approaches for solving soil-structure interaction problems. Since 1990 s, great effort has been made for substituting the classical 
methods of design by the new ones based on the concept of performance-based seismic design. Performancebased engineering (PBE) is a technique for seismic evaluation and design using performance level prediction for safety and risk assessment. Development of this approach has been a natural outgrowth of the evaluation and upgrade process for existing buildings. Performance objectives are expressed as an acceptable level of damage, typically categorised as one of several performance levels. Performance levels describe the state of structures after being subjected to a certain hazard level and are classified as: fully operational, operational, life safe, near collapse, or collapse. Overall lateral deflection, ductility demand, and inter-storey drifts are the most commonly used damage parameters. The above mentioned five qualitative performance levels are related to the corresponding quantitative maximum inter-storey drifts of: $0.2 \%, 0.5 \%, 1.5 \%$, $2.5 \%$, and $>2.5 \%$, respectively.

Soil-structure interaction particularly for un-braced structures resting on relatively soft soils may significantly amplify the lateral displacements and interstorey drifts. This amplification of lateral deformations may change the performance level of the building frames. Thus, a comprehensive dynamic analysis to evaluate the realistic performance level of a structure should consider effects of SSI in the model. In addition, the necessity of estimating the vulnerability of existing structures and assessing reliable methods for their retrofit have greatly attracted the attention of engineering community in most seismic zones throughout the world. Although several researchers (e.g. Krawinkler et al. 2003; Galal and Naimi 2008; Tabatabaiefar and Massumi 2010) have studied structural behaviour of building structures subjected to earthquake under the influence of soil-structure interaction conducting numerical simulations, the accuracy and integrity of only a few of the utilised numerical models were examined and validated against laboratory based experimental test results. Therefore, further verification of the numerical models is required to ensure the predictions are in a good agreement and conformity with measurements.

The problem of soil-structure interaction in the seismic analysis and design of structures has become increasingly important, as it may be inevitable to build structures at locations with less favourable geotechnical conditions in seismically active regions. The Mexico City earthquake in 1985 and Christchurch earthquake in 2011 (New Zealand) clearly illustrate the importance of local soil properties on the earthquake response of structures. These earthquakes demonstrated that the ground motions could be significantly amplified at the base of the structure. Trifunac (1990) showed that for high frequencies, the amplification effects compete with an elastic attenuation. For long periods, along the period axis, the amplification can be described by a low-pass filter with roll-off near the period for which the quarter wavelength of wave motion in soil, or in sediments, coincides with their thickness. In the intermediate period range the amplification depends only on the impedance jump between the 'soft' surface materials and 'hard' basement, and is independent of the thickness of soil or of sedimentary layers. In addition, some theoretical investigations (e.g. Wolf 1985; Bi and Hao 2012) have clearly highlighted the importance of the local soil amplification effect. These two references are suggested to be added. Therefore, there is a need to design structures safely but not costly against natural disasters such as earthquakes. Effects of dynamic soilstructure interaction under extreme loads due to strong earthquakes are significant for many classes of structures. Consequently, an accurate assessment of the inertial forces and displacements in structures requires a rational treatment of SSI effects. In this study, an enhanced numerical soil-structure model has been developed which treats the behaviour of soil and structure with equal rigor. The proposed numerical soilstructure model has been verified and validated by performing experimental shaking table tests. In addition, effects of soil-structure interaction on the seismic response of a moment resisting building frame have been experimentally investigated.

\section{DEVELOPED NUMERICAL SOIL- STRUCTURE MODEL}

The governing equations of motion for the structure incorporating foundation interaction and the method of solving these equations are relatively complex. Therefore, direct method, the method in which the entire soil-structure system is modelled in a single step, is employed in this study. The soil-structure system simulated adopting direct method, composed of structure, common nodes, soil foundation system and earthquake induced acceleration at the level of the bedrock, is shown in Figure 1. The dynamic equation of motion of the soil and structure system can be written as:

$$
[M]\{\ddot{u}\}+[C]\{\dot{u}\}+[K]\{u\}=-[M]\{a\} \ddot{u}_{g}+\left\{F_{v}\right\}
$$

where, $\{u\},\{\dot{u}\}$, and $\{\ddot{u}\}$ are the nodal displacements, velocities and accelerations with respect to the underlying soil foundation, respectively. $[M],[C]$ and $[K]$ are the mass, damping, and stiffness matrices of the structure, respectively. It is more appropriate to use the 


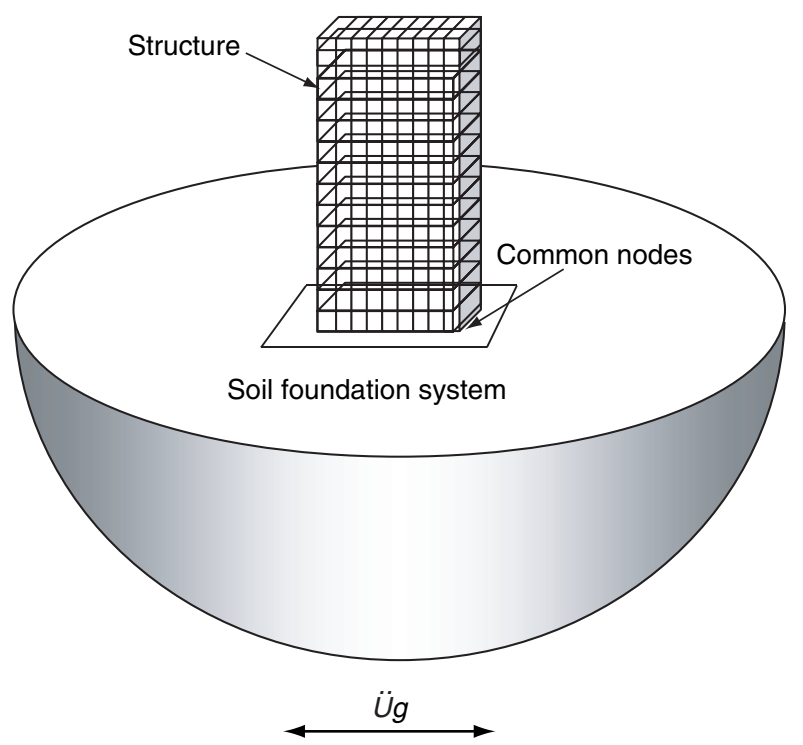

Figure 1. Soil-structure system in direct method

incremental form of Eqn 1 when plasticity is included, and then the matrix $[K]$ should be the tangential matrix and $\left\{\ddot{u}_{g}\right\}$ is the earthquake induced acceleration at the level of the bedrock. For example, if only the horizontal acceleration is considered, then $\{a\}=[1,0,1,0, \ldots .1$, $0]^{T} .\left\{F_{v}\right\}$ is the force vector corresponding to the viscous boundaries. This vector is nonzero only when there is a difference between the motion on the near side of the artificial boundary and the motion in the free field (Wolf 1985).

To model soil-structure system in direct method, a novel and enhanced soil-structure model is developed in FLAC2D to simulate various aspects of complex dynamic soil-structure interaction in a realistic and rigorous manner. FLAC 2D (Fast Lagrangian Analysis of Continua) is a two-dimensional explicit finite difference program for engineering mechanics computations. This program can simulate the behaviour of different types of structures. Materials are represented by elements which can be adjusted to fit the geometry of the model. Each element behaves according to a prescribed linear or nonlinear stress/strain law in response to the applied forces or boundary restraints.

Seo et al. (2007) developed three-dimensional frequency-dependent elements for soil-structure interaction analysis and compared the analytical results of their 3D model with three other 2D plane strain models from the past studies. They indicated that although good results were obtained using the 3D elements, there were limitations to deal with structures having complex geometries and 2D plane strain predictions are in good agreement with three dimensional results. The real building models utilised in this study are assumed to be long in perpendicular direction. Therefore, plane strain assumption is valid and 3D effects are negligible. Thus, each frame model used in $2 \mathrm{D}$ modelling procedure represents the same attributes as the entire parallel building frames in perpendicular direction.

The soil-structure model employs beam structural elements to model beams, columns and foundation slabs. During analysis process, structural material could behave as an isotropic, linearly elastic material with no failure limit for elastic structural analysis or as an elastic-perfectly plastic material with a specified limiting plastic moment for inelastic structural analysis. Therefore, both elastic and plastic (inelastic) structural behaviour can be captured by the model in dynamic analysis. In addition, structural geometric nonlinearity (large displacements) has been accommodated in dynamic analysis. Two dimensional plane-strain grids composed of quadrilateral elements are utilised to model the soil medium. Nonlinear behaviour of the soil medium has been captured using backbone curves of shear modulus ratio versus shear strain $\left(G / G_{\max }-\gamma\right)$ and damping ratio versus shear strain $(\xi-\gamma)$ adopting MohrCoulomb failure model. Employing the backbone curves for simulating nonlinear behaviour of the soil, in this study, fully nonlinear method for analysis of dynamic soil-structure interaction has been employed in order to attain rigorous and reliable results. Fully nonlinear method is capable to precisely model nonlinearity in dynamic analysis of soil-structure systems and follow any prescribed nonlinear constitutive relation. It should be noted that earthquake ground motions adopted in this study are horizontal acceleration records of four benchmark earthquakes including Northridge (1994), Kobe (1995), El Centro (1940) and Hachinohe (1968). The adopted horizontal acceleration records are applied to the base of the soilstructure model at the level of the bedrock.

The foundation facing zone in numerical simulations is separated from the adjacent soil zone by interface elements to simulate frictional contact. Interface elements are mainly used to transfer the structural reactions to the subsoil beneath the structure, and simulate frictional contact and probable slip due to seismic excitation. The interface between the foundation and soil is represented by a normal and shear stiffness between two planes contacting each other and is modelled as linear spring-slider systems, with interface shear strength defined by the Mohr-Coulomb failure criterion. The relative interface movement is controlled by interface stiffness values in the normal and tangential directions. It should be noted that shear strength of the interface has been simulated using Mohr-Column model, assuming that there is no slip in the interface until the 
shear strength of the interface is reached and after that perfectly plastic deformation would occur. The normal and shear springs control the force transfer process from one surface to the other surface. This is a simplifying assumption that has been only used for interface modelling. Since the shear strength of the interface has not been reached under the applied loads, there has been insignificant slip between the soil and foundation, and thus this assumption does not influence the numerical results. Turan et al. (2013) stated that, considering embedment depth in soil-structure interaction analysis, the structural response amplitude slightly decreases and the resonance frequency becomes a bit higher with increasing embedment depth. In addition, it should be noted that although the embedment depth of the foundation may influence the ultimate results of the numerical analyses (e.g. Trifunac et al. 2001a ; Turan et al. 2013 ), for a conservative design and analysis, it is assumed that the foundations of the structural models are located close to the ground surface.

The interface between the foundation and soil is represented by normal $\left(k_{n}\right)$ and shear $\left(k_{s}\right)$ springs between two planes contacting each other and is modelled using linear spring system, with the interface shear strength defined by the Mohr-Coulomb failure criterion (Figure 2). The relative interface movement is controlled by interface stiffness values in the normal and tangential directions. Normal and shear spring stiffness values for interface elements of the soil-structure model are set to ten times the equivalent stiffness of the neighbouring zone based on recommended relationship by Rayhani and EL Naggar (2008) and Itasca Consulting Group (2008) for the isotropic soil medium as follows:

$$
k_{s}=k_{n}=10\left[\frac{K+\frac{4}{3} G}{\Delta z_{\min }}\right]
$$

where, $K$ and $G$ are bulk and shear modulus of neighbouring zone, respectively, and $\Delta z_{\min }$ is the smallest width of an adjoining zone in the normal direction. This is a simplifying assumption that has been only used for interface modelling. Since there is no large slip between the soil and foundation in this study, this assumption does not influence the numerical results. The strip reinforced concrete foundation is modelled using "Beam Structural Elements" being 4 meters wide, 12 meters long and, 1 meter deep. Beam Structural Elements are two-node, straight, finite elements with six degrees of freedom per node comprising three translational and three rotational components. Therefore, the mentioned elements can deform in the vertical direction simulating concrete footing flexibility as the modelling is conducted in plane-strain condition, the effective strip foundation width has been taken into account to calculate the moment of inertia of the concrete raft foundation. For lateral boundaries of the soil medium, viscous boundaries (quiet boundaries) proposed and developed by Lysmer and Kuhlemeyer (1969) are utilised in this study. The proposed method is based on utilisation of independent dashpots in the normal and shear directions at the model boundaries. The dashpots provide viscous normal and shear tractions given by

$$
\begin{gathered}
T_{n}=-\rho \cdot C_{p} \cdot v_{n} \\
T_{s}=-\rho \cdot C_{s} \cdot v_{s}
\end{gathered}
$$

where, $T_{n}$ and $T_{s}$ are normal and shear tractions at the model boundaries, respectively; $v_{n}$ and $v_{s}$ are the normal and shear components of the velocity at the boundary respectively; $\rho$, is the material density; and $C_{p}$ and $C_{s}$ are the $p$-wave and $s$-wave velocities, respectively. It should be noted that the utilised boundary conditions in this study, have been previously used by other

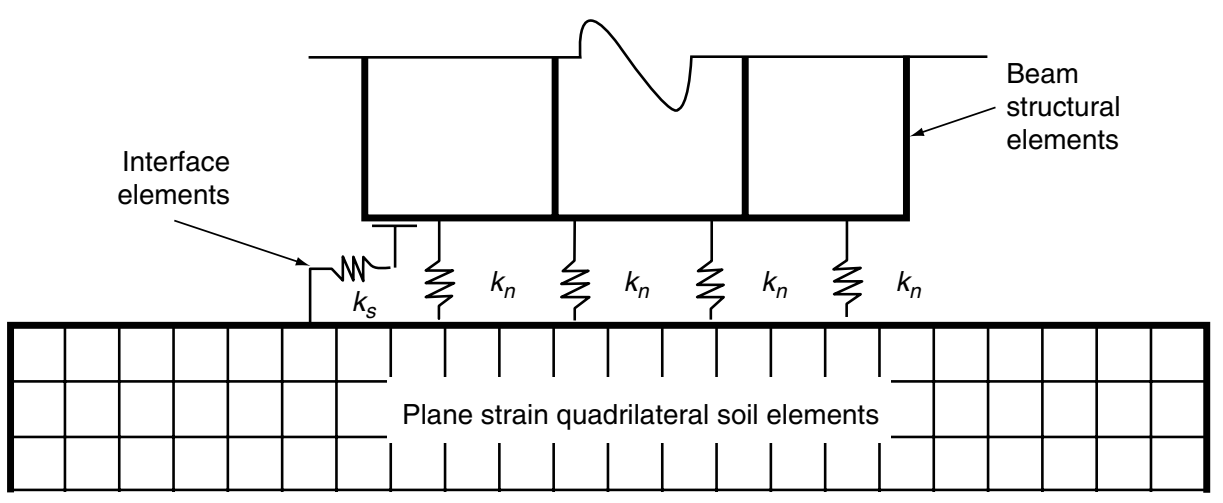

Figure 2. Interface elements including normal $\left(k_{n}\right)$ and shear $\left(k_{s}\right)$ springs 
researchers (e.g. Beaty and Byrne 2001; Byrne 2006) for dynamic soil-structure interaction modelling.

In the developed soil-structure model in this study, the boundary conditions at the sides of the model account for the free-field motion which would exist in the absence of the structure. Free-field boundaries have been simulated using a developed technique, involving the execution of a one-dimensional free-field calculation in parallel with the main-grid analysis. Thus, plane waves propagating upward undergo no distortion at the boundaries because the free-field grid supplies conditions identical to those in an infinite model. The components of the soil-structure model are illustrated in Figure 3.

Simple methods such as Winkler computational model are often used in engineering practice in which soil-structure interaction is modelled using either linear or non-linear springs. The reliability of these constitutive models has been questioned by many due to the simplifying assumptions regularly used (e.g. Ashour et al. 1998). As mentioned by Chu (20006) for systems with strong nonlinear behaviour, coupled soil-structure response analysis is highly desirable which can explicitly express the relationship between the soil and the structural responses. The new developed model is a novel and enhanced numerical soil-structure model as it is capable of capturing structural plasticity (inelastic behaviour) and soil nonlinearity, treating the behaviour of both soil and structure with equal rigor simultaneously. Besides, adopting direct method, which perfectly simulates complex geometries and material properties in numerical methods, the model can perform fully nonlinear time history dynamic analysis to simulate realistic dynamic behaviour of the soil and the structure under seismic excitations as accurate and realistic as possible. In addition, as the model employs a Multi Degree of Freedom (MDOF) structure, interstorey drifts can be determined and utilised for investigating the performance levels of the building structures under the influence of dynamic soil-structure interaction. It should be noted that none of the numerical models utilised in the past numerical soil-structure interaction investigations (e.g. Krawinkler et al. 2003; Galal and Naimi 2008; Koutromanos et al. 2009; Tabatabaiefar and Massumi 2010; Tavakoli et al. 2011) have adopted the novel and enhanced combined capabilities of the new developed numerical soilstructure model.

\section{SHAKING TABLE EXPERIMENTAL TESTS}

Full-scale field tests or scale model tests are essential to study soil-structure system behaviour during earthquakes. Such tests are also required to validate numerical or analytical models. According to Trifunace et al. (2001b) and Pitilakis et al. (2013), experimental studies of soil-structure interaction are best conducted in full-scale using periodic force excitation of structures. Full-scale field experiments have the advantage of

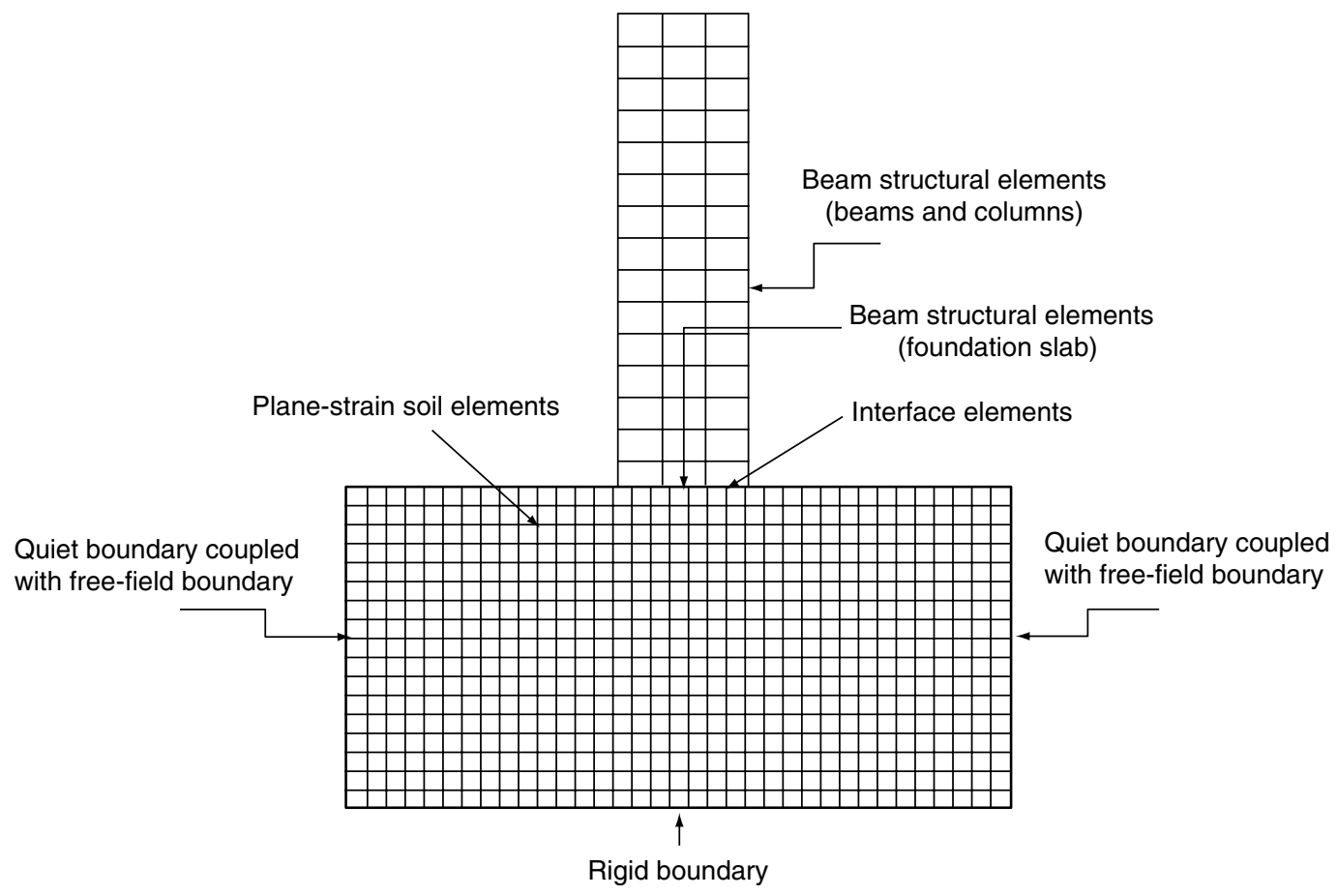

Figure 3. Components of the soil-structure model 
considering realistic site conditions, whereas, the use of scale models on shaking table offers the advantage of simulating complex systems under controlled conditions, and the opportunity to gain insight into the fundamental mechanisms operating in these systems. In many circumstances, the scale models on shaking table may afford a more economical option than the corresponding full-scale tests. The practice of conducting parametric studies with scale models can be used to augment areas where case histories and prototype tests provide only sparse data. In addition to qualitative interpretation, scale model test results are often used as calibration benchmarks for analytical methods, or to make quantitative predictions of the prototype response.

For such applications, it is necessary to have a set of scaling relations which can relate the observations and predictions. Shaking table test is an experimental technique used in earthquake engineering to simulate ground motions. Since the emergence of shaking tables in the $1920 \mathrm{~s}$, large number of earthquake model tests have been performed. Shaking table tests have been considered as $1 g$ modelling, in which the gravity acceleration of the model and prototype are always the same. Shaking table test is relatively cheap and easy to model complex prototypes, although there is a lack of accuracy due to $1 g$ manner (e.g. low confining pressure of model affects test results especially in sandy soils). It should be noted that, in centrifuge tests by increasing the gravity force via rotating the model, it is possible to accurately model the soil stress- strain condition as exists in prototype. In comparison, although centrifuge test models the stress-strain conditions accurately, it is difficult to build complex prototypes, and due to small size of the model, fewer instruments can be installed (Taylor 1997).

During the past few decades, several researchers have carried out shaking table tests on soil-structure systems using various types of soil containers and structural models. In many of the past experiments, the structure model on top of the soil has not been taken into consideration at all. Some of the tests were only performed on the soil inside the container (e.g. Taylor 1997; Prasad et al. 2004; Lee et al. 2012) in order to investigate dynamic behaviour of the soil under the influence of earthquake loads, while some others were undertaken on soil-foundation system to observe the dynamic interaction of shallow or pile foundation with the underlying soil (e.g. Stanton et al. 1998; Richards et al. 1990). In some of the past mentioned experiments, the structural model has been considered but simplified to SDOF (single Degree of Freedom) oscillator (e.g. Meymand 1998; Ishimura et al. 1992; Jakrapiyanun
2002; Pitilakis et al. 2008; Chau et al. 2009) so as to model and investigate dynamic soil-structure interaction. However, by simplifying the structural model, the behaviour of the soil-structure system may not be completely conforming to reality.

Unlike past shaking table experiments which were performed without the structure or employed simplified SDOF (single Degree of Freedom) oscillators, in this study, the adopted structural model will simulate most of the structural properties of the real prototype building such as frequency of vibrations, number of stories, and mass. Therefore, this experiment will be a unique experimental shaking table test considering the structural model in the soil-structure system precisely. As a result, realistic seismic response of a multi-storey frame could be determined experimentally and compared with the numerical modelling results.

As mentioned earlier, in this study, the proposed numerical soil-structure model has been validated and verified by performing shaking table tests to the scale soil-structure model. The dynamic simulation has been carried out on the shaking table located in the structures laboratory of the University of Technology Sydney (UTS). It should be noted that UTS shaking table has a uni-axial configuration, allowing for one-dimensional input motions. The shaking table is $3 \mathrm{~m} \times 3 \mathrm{~m}$ table with testing frequency range between 0.1 to $50 \mathrm{HZ}$, maximum payload of 10 tonnes, and overturning moment of $100 \mathrm{kN}-\mathrm{m}$.

\section{PROTOTYPE CHARACTERISTICS}

The prototype of the experimental tests is a soilstructure system with dimensional characteristics illustrated in Figure 4. The prototype building frame of the soil-structure system is a fifteen storey concrete moment resisting frame. The building frame height and width are 45 and 12 metres, respectively and spacing between the frames into the page is 4 metres. The building is resting on a footing which is 4 meters wide and 12 meters long. Natural frequency of the prototype building is $0.384 \mathrm{~Hz}$ and its total mass is 953 tonnes. Soil medium underneath the structure is a clayey soil with shear wave velocity of $200 \mathrm{~m} / \mathrm{s}$ and unit weight of $14.40 \mathrm{kN} / \mathrm{m}^{3}$ (soil density of $1470 \mathrm{~kg} / \mathrm{m}^{3}$ ). The horizontal distance of the soil lateral boundaries and bedrock depth have been selected to be 60 metres and 30 metres, respectively.

\section{SCALING FACTORS FOR SHAKING TABLE TESTING}

Scale models can be defined as having geometric, kinematic, or dynamic similarities to the prototype (Langhaar 1951; Sulaeman 2010). Geometric similarity 


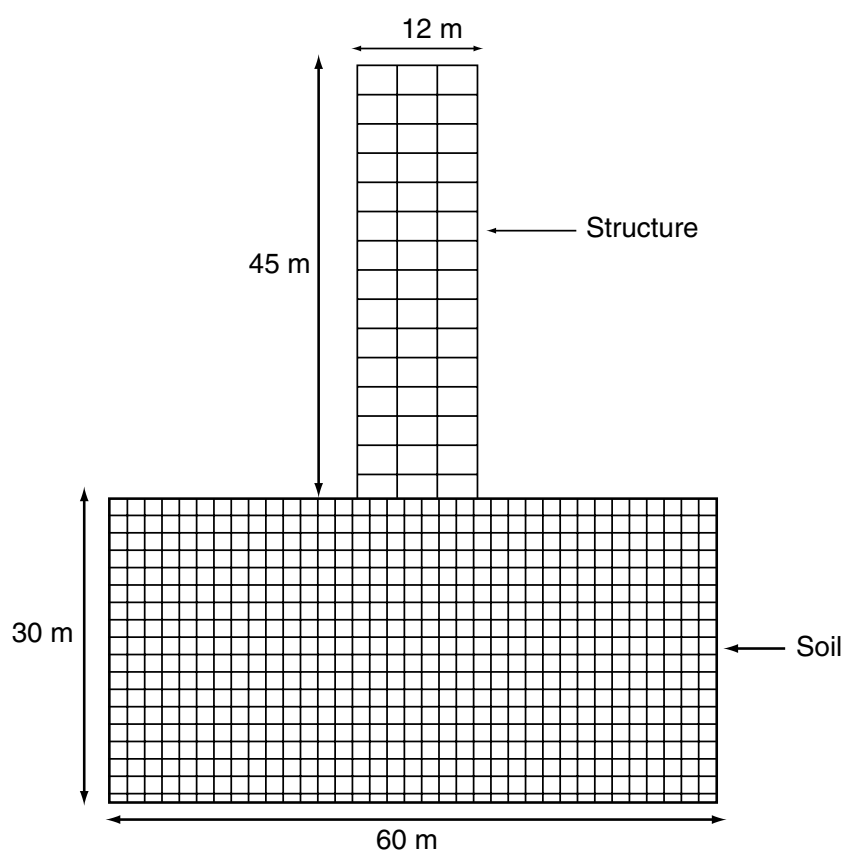

Figure 4. Dimensional characteristics of the prototype

defines a model and prototype with homologous physical dimensions. Kinematic similarity refers to a model and prototype with homologous particles at homologous points at homologous times. Dynamic similarity describes a condition where homologous parts of the model and prototype experience homologous net forces. The objective of the scale modelling procedure for this test program is to achieve "dynamic similarity", where model and prototype experience homologous forces. For this purpose, adopted methodology by Meymand (1998) is the framework for scale model similitude in this study. According to this approach, three principal test conditions establish many of the scaling parameters. The first condition is that testing is conducted in 1-g environment, which defines model and prototype accelerations to be equal. Secondly, a model with similar density to the prototype is desired, fixing another component of the scaling relations. Thirdly, the test medium is primarily composed of saturated clayey soil, whose undrained stress-strain response is independent of confining pressure, thereby simplifying the constitutive scaling requirements. In addition to the three principal test conditions, Meymand (1998) pointed out that the natural frequency of the prototype should be scaled by an appropriate scaling relation. By defining scaling conditions for density and acceleration, the mass, length, and time scale factors can all be expressed in terms of the geometric scaling factor $(\lambda)$, and a complete set of dimensionally correct scaling relations (ratio of prototype to model) can be derived for all variables being studied. The scaling relations for the
Table 1. Scaling relations in terms of geometric scaling factor $(\lambda)$

\begin{tabular}{lccccc}
\hline Mass Density & 1 & Acceleration & 1 & Length & $\lambda$ \\
Force & $\lambda^{3}$ & Shear Wave Velocity & $\lambda^{1 / 2}$ & Stress & $\lambda$ \\
Stiffness & $\lambda^{2}$ & Time & $\lambda^{1 / 2}$ & Strain & 1 \\
Modulus & $\lambda$ & Frequency & $\lambda^{-1 / 2}$ & EI & $\lambda^{5}$ \\
\hline
\end{tabular}

variables contributing to the primary modes of system response, adopted in this study, are shown in Table 1. The mentioned scaling relations have been utilised by many researchers (e.g. Meymand 1998; Turan et al. 2009; Moss et al. 2010; Sulaeman 2010; Lee et al. 2012) in soil-structure interaction shaking table test experiments.

Adopting an appropriate geometric scaling factor $(\lambda)$ is one of the important steps in scale modelling on shaking table. Although small scale models could save cost, the precision of the results could be substantially reduced. Considering the specifications of UTS shaking table, scaling factor of 1:30 provides the largest achievable scale model with rational scales, maximum payload, and overturning moment which meet the facility limitations. Thus, geometric scaling factor $(\lambda)$ of 1:30 is adopted for experimental shaking table tests on the scale model in this study.

\section{SOIL-STRUCTURE MODEL COMPONENTS}

In this study, soil-structure model possesses three main components including the structural model, the laminar soil container, and the soil mix. Details and characteristics of these components are explained below.

\subsection{Structural Model}

Employing geometric scaling factor of $1: 30$, as explained above, height, length, and width of the structural model are determined to be, $1.50 \mathrm{~m}, 0.40 \mathrm{~m}$, and $0.40 \mathrm{~m}$, respectively. As explained above, in addition to geometric dimensions, the natural frequency of the prototype should be scaled by an appropriate scaling relation and the density of the model and the prototype should be equal. In this way, prototype structure can be modelled more accurately in shaking table tests. According to Table 1, the scaling relationship between natural frequency of the model $\left(f_{m}\right)$ and natural frequency of the prototype $\left(f_{p}\right)$ is:

$$
\frac{f_{m}}{f_{p}}=\frac{1}{\sqrt{\lambda}}=5.48
$$

As mentioned in Section 4, natural frequency of the prototype structure is $f_{p}=0.384 \mathrm{~Hz}$. Therefore, with 
respect to Eqn 5, the required natural frequency of the structural model $\left(f_{m}\right)$ is $2.11 \mathrm{~Hz}$. Furthermore, based on scaling relationship on Table 1 , the density of the model $\left(\rho_{m}\right)$ should be equal to the density of the prototype $\left(\rho_{p}\right)$. Density of the prototype structure $\left(\rho_{p}\right)$ can be determined as follows:

$$
\rho_{p}=\frac{m_{p}}{V_{p}}=\frac{953,000}{45 \times 12 \times 4}=441 \mathrm{~kg} / \mathrm{m}^{3}
$$

where, $m_{p}$ is the mass of the prototype structure and $V_{p}$ is the volume of the prototype structure. Therefore, the mass of the structural model $\left(m_{\mathrm{m}}\right)$ can be estimated as:

$$
\begin{aligned}
m_{m} & =\rho_{m} \times V_{m}=441 \mathrm{~kg} / \mathrm{m}^{3} \\
& \times(1.50 \mathrm{~m} \times 0.40 \mathrm{~m} \times 0.40 \mathrm{~m})=106 \mathrm{k}
\end{aligned}
$$

where, $V_{m}$ is the volume of the structural model. Based on the above mentioned discussion, the required characteristics of the structural model are summarised in Table 2. Knowing the required characteristics of the structural model, its 3D numerical model has been built in SAP2000 software using two dimensional shell elements to model columns and floors as shown in Figure 5. The numerical model consists of fifteen horizontal steel plates as the floors and four vertical steel plates as the columns. Steel plate grade 250, according to AS/NZS 3678-2011 (Structural Steel), with the minimum yield stress of $280 \mathrm{MPa}$ and minimum tensile strength of $410 \mathrm{MPa}$, has been adopted in the design. The thickness of the steel plates have been determined in design process after several cycles of trial and error in order to fit the required natural frequency and mass as summarised in Table 2. The finalised base plate is a $500 \times 500 \times 10 \mathrm{~mm}$ steel plate while the floors consist of $400 \times 400 \times 5 \mathrm{~mm}$ plates and four $500 \times 40 \times$ $2 \mathrm{~mm}$ steel plates are used for the columns. The connections between the columns and floors are provided using stainless steel metal screws with $2.5 \mathrm{~mm}$ diameter and $15 \mathrm{~mm}$ length. After the numerical modelling and design, the structural model was constructed in house. The completed structural model is shown in Figure 6. The mass of the model $\left(m_{m}\right)$, without the base plate, was measured to be $104 \mathrm{~kg}$ which matches the required structural mass (Table 2). Total measured mass of the structural model considering the mass of the base plate is $115 \mathrm{~kg}$.

\subsection{Laminar Soil Container}

The geotechnical model cannot be directly mounted on shake table because of the requirements of confinement. To model the soil in shaking table tests, a container is required to hold the soil in place. During the past few decades, several studies have been conducted on soilstructure systems using various types of soil containers. Many researchers (e.g. Gazetas 1982; Taylor et al. 1995; Pitilakis et al. 2008; Tang et al. 2009; Cheung et al. 2013) concluded that laminar soil containers are the most appropriate and efficient type of the soil containers. Based on the conclusions made by the above mentioned researchers, well designed laminar soil containers can better model the free field boundary conditions in comparison with rigid and flexible containers as the lateral deformations in laminar soil containers are almost identical to the free field movements. According to Turan et al. (2009) and Qin

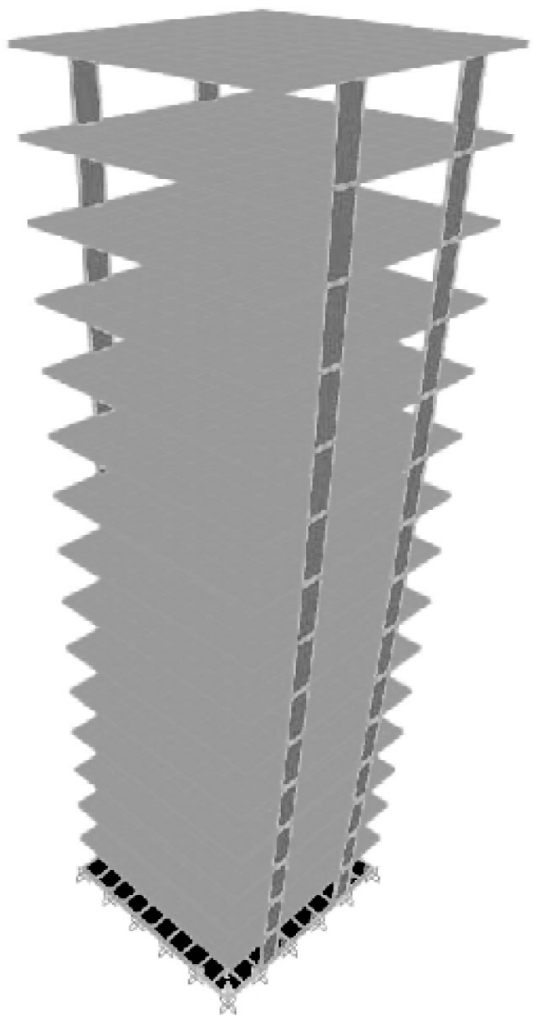

Figure 5. 3D numerical model of the structural model in SAP2000

Table 2. Required characteristics of the structural model

\begin{tabular}{lcccc}
\hline Total height $(\mathbf{m})$ & Total length $(\mathbf{m})$ & Total width $(\mathbf{m})$ & Natural frequency $(\mathbf{H z})$ & Total mass $(\mathbf{k g})$ \\
\hline 1.50 & 0.40 & 0.40 & 2.11 & 106 \\
\hline
\end{tabular}




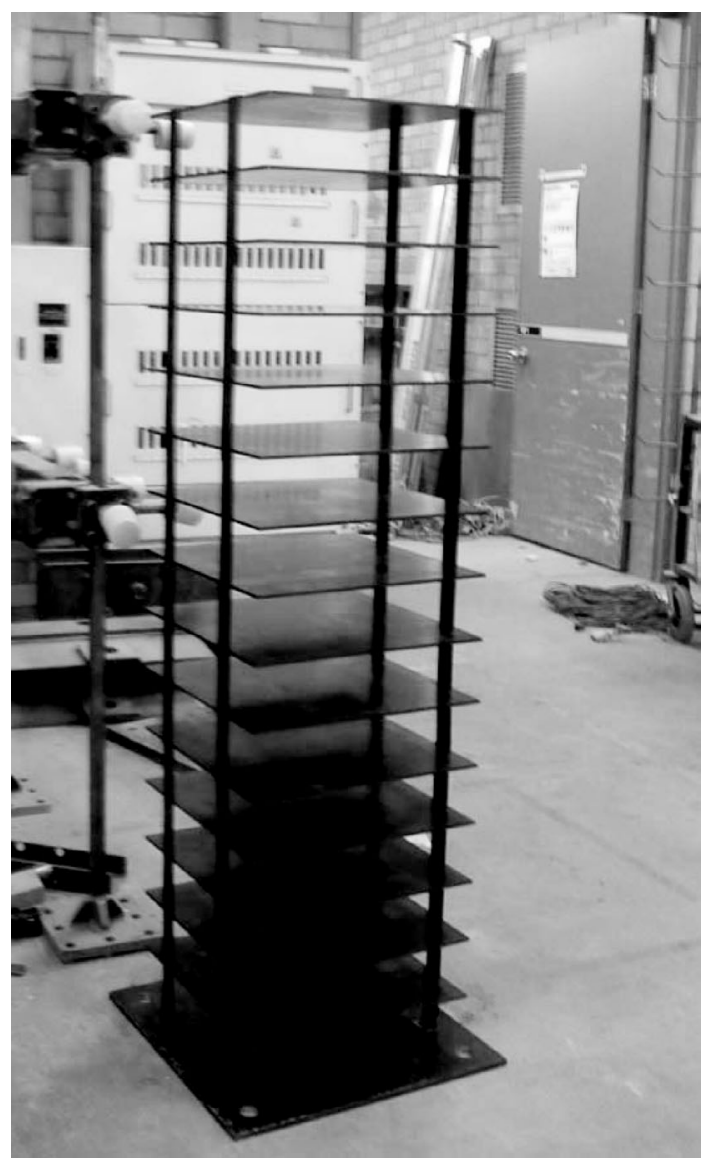

Figure 6. Completed structural model

et al. (2013), the laminar soil container does not impose significant boundary effects and is able to maintain 1-D soil column behavior. In addition, they concluded that the dynamic behavior of the soil in the laminar soil container during scaled model tests is consistent with the behavior measured during cyclic laboratory tests. In addition, lateral motion of the entire depth of a laminar soil container follows the sinusal shape which represents authentic conditions of the free field ground motion. Therefore, in order to perform rigorous and reliable experimental shaking table tests, a laminar soil container has been employed in this study.

By selecting 1:30 as the geometric scaling factor, the container should have minimum length, width, and depth of $2.0 \mathrm{~m}, 1.20 \mathrm{~m}$, and $1.0 \mathrm{~m}$, respectively. Allowing a further $10 \mathrm{~mm}$ on each side for construction purposes similar to Prasad et al. (2004), the final length, width, and depth of the laminar soil container are estimated to be $2.10 \mathrm{~m}, 1.30 \mathrm{~m}$, and $1.10 \mathrm{~m}$, respectively. In terms of choosing the materials to build the soil container, according to the previous conducted research works (e.g. Ishimura et al. 1992; Taylor 1997; Jakrapiyanun 2002; Pitilakis et al. 2008; Chau et al. 2009), aluminium frames and rubber layers were

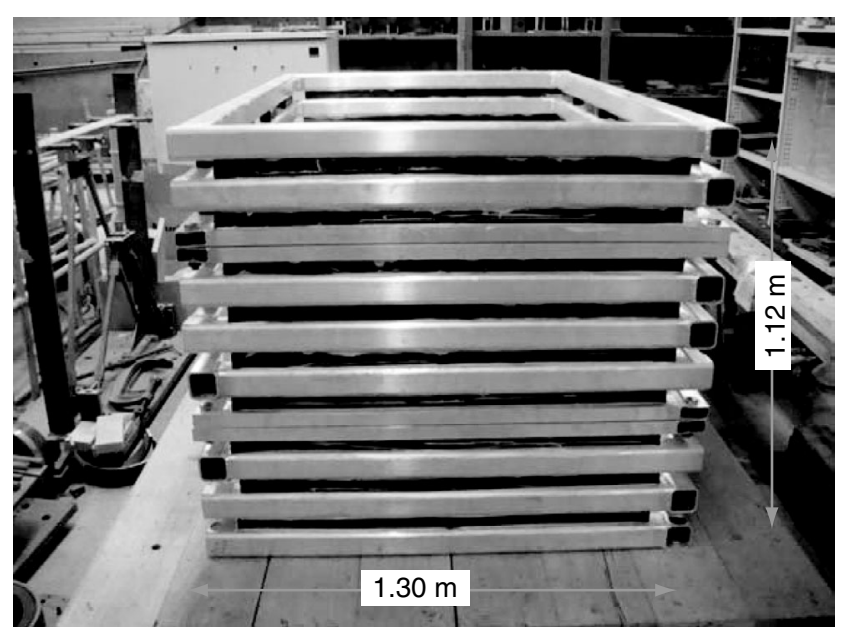

Figure 7. Laminar soil container view constructed in the UTS structures laboratory

employed in an alternating pattern. Therefore, the laminar soil container consists of a rectangular laminar box made of aluminium rectangular hollow section frames separated by rubber layers. The aluminium frames provide lateral confinement of the soil, while the rubber layers allow the container to deform in a shear beam manner. The employed laminar soil container in this study, constructed in house, is shown in Figure 7. The natural frequency of the laminar soil container was measured to be $10 \mathrm{~Hz}$ in the laboratory and it was noted that it is equal to the required natural frequency.

\subsection{Soil Mix}

In this study, a synthetic clay mixture was adopted as the soil medium for the shaking table testing process. In order to develop the synthetic clay mixture, Q38 kaolinite clay, ActiveBond 23 bentonite, class F fly ash, lime, and water were used as the components of the soil mixture. The proposed mix was prepared three times to control repeatability of the test and each time three cylindrical test specimens of size $D=50 \mathrm{~mm}$ and $h=100 \mathrm{~mm}$ were taken. To measure shear wave velocity of the mix over the cure age, bender element tests were performed. The soil specimens were placed between bender elements, and shear wave velocity of each soil specimen was obtained at different cure ages. Figure 8 shows the measured shear wave velocity of the soil mix at different cure ages. According to Figure 8, the soil mix produces the required shear wave velocity of $36 \mathrm{~m} / \mathrm{s}$ (based on the scaling factor in Table 1) on the second day of its cure age. Afterwards, the standard method of soil density determination was performed on the second day of the cure age according to AS 1289.3.5.1-2006 (Methods of testing soils for engineering purposes). Accordingly, soil density in the second day of the cure 


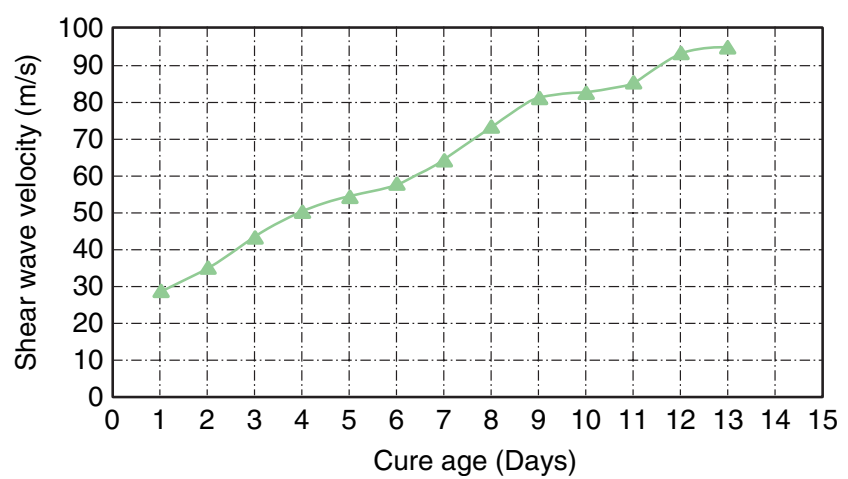

Figure 8. Shear wave velocities versus cure age for the examined $\operatorname{mix}$

age $\left(\rho_{s}\right)$ was determined to be $1450 \mathrm{~kg} / \mathrm{m}^{3}$ which is almost equal to the prototype soil density $\left(1470 \mathrm{~kg} / \mathrm{m}^{3}\right)$. Thus, shear wave velocity and soil density values of produced soil mix on the second day of the cure age satisfy the dynamic similarity requirements, explained in Section 5. In addition, in order to ensure that the soil undrained shear strength is adequate to satisfy the required shallow foundation bearing capacity underneath the structural model, three cylindrical test specimens of size $D=100 \mathrm{~mm}$ and $h=200 \mathrm{~mm}$ were taken from the soil mix. Then, two days of curing, Unconfined Compression tests (UC) were performed on the three soil specimens in accordance with AS5101.4-2008 (Method 4: unconfined compressive strength of compacted materials) in order to determine the soil shear strength. The average undrained shear strength $\left(S_{u}\right)$ of the mix on the second day of the cure age, resulting from three examined specimens, was $1.57 \mathrm{kPa}$. According to the carried out foundation calculations, by adopting this value of undrained shear strength, the soil mix will provide enough bearing capacity with acceptable factor of safety under the structural model on the second day of the cure age to avoid any failure or excessive settlement underneath the structure $\left(q_{u l t}=5.14 S_{u}\right.$, FOS $>2.0$, where $q_{u l t}$ is the ultimate bearing capacity of the foundation and FOS is factor of safety). Eventually, the soil mix on the second day of the cure age is expected to have the properties summarised in Table 3.

Table 3. Properties of the selected soil mix on the second day of cure age

\begin{tabular}{lcc}
\hline $\begin{array}{l}\text { Shear wave } \\
\text { velocity } \\
\boldsymbol{V}_{\boldsymbol{s}}(\mathbf{m} / \mathbf{s})\end{array}$ & $\begin{array}{c}\text { Undrained shear } \\
\text { strength } \\
\boldsymbol{S}_{\boldsymbol{u}}(\mathbf{k P a})\end{array}$ & $\begin{array}{c}\text { Soil density } \\
\rho\left(\mathbf{k g} / \mathbf{m}^{3}\right)\end{array}$ \\
\hline 36 & 1.57 & 1450 \\
\hline
\end{tabular}

\section{SCALING OF ADOPTED EARTHOUAKE ACCELERATION RECORDS}

Four earthquake acceleration records including Kobe, 1995 [Figure 9(a)], Northridge, 1994 [Figure 10(a)], El Centro, 1940 [Figure 11(a)], and Hachinohe, 1968 [Figure 12(a)] have been adopted for the shaking table tests. The first two earthquakes are near field ground motions and the latter two are far field motions. These earthquakes have been chosen by the International Association for Structural Control and Monitoring for benchmark seismic studies (Karamodin and Kazemi 2008). Characteristics of the mentioned earthquake ground motions are summarised in Table 4. According to Table 1 and as determined by Eqn 3, scaling relationship between natural frequency of the model $\left(f_{m}\right)$

(a)

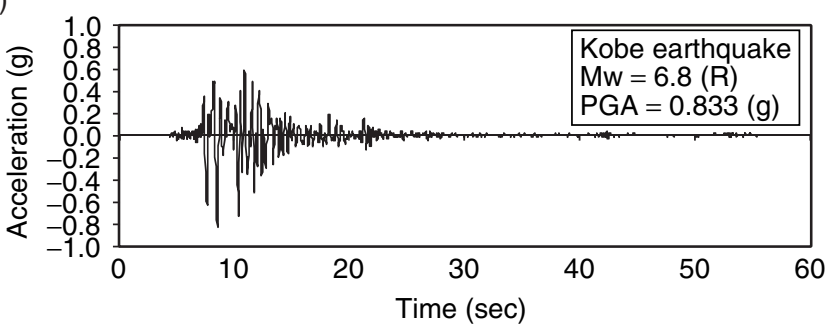

(b)

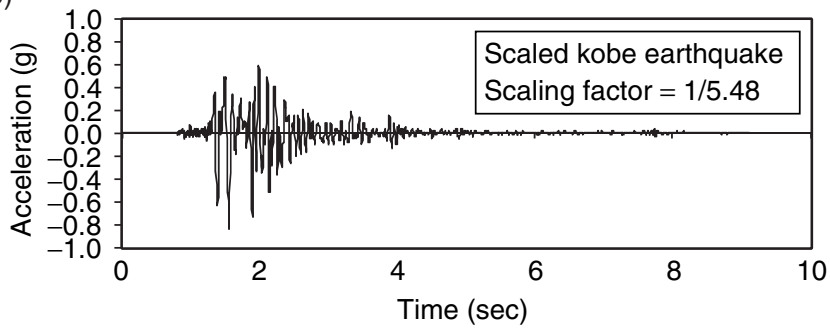

Figure 9. Kobe earthquake (1995): (a) original record; (b) scaled record
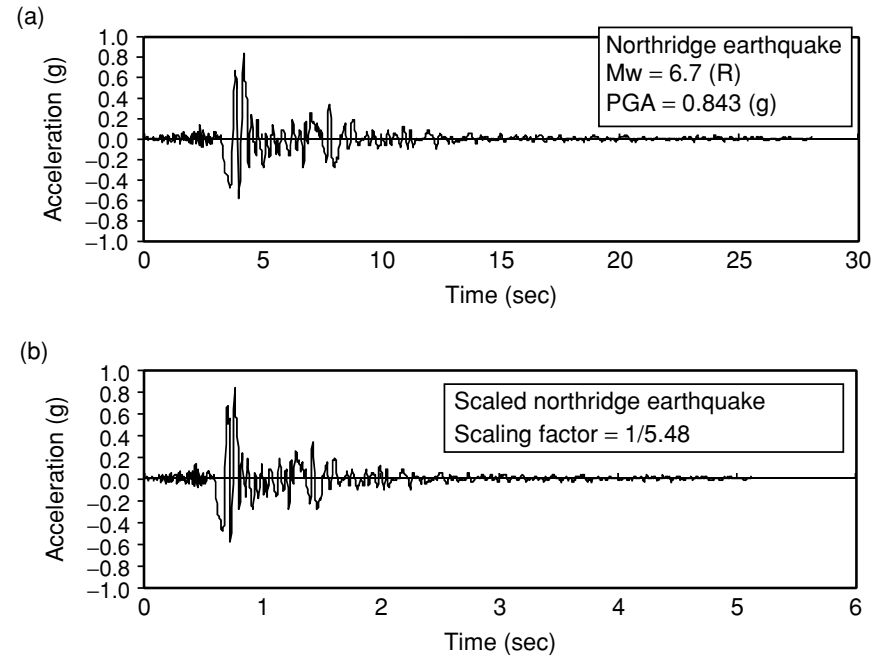

Figure 10. Northridge earthquake (1994): (a) original record; (b) scaled record 
(a)

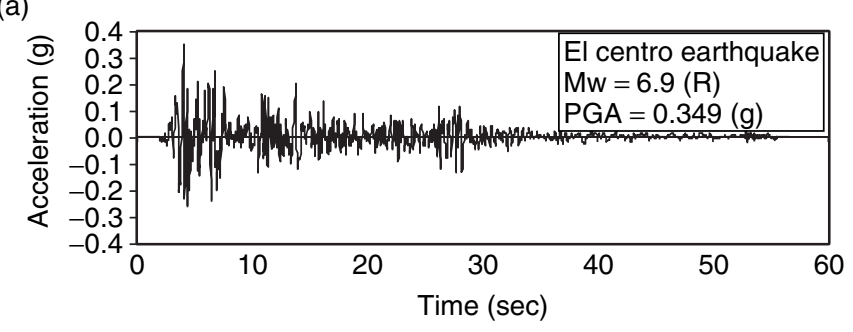

(b)

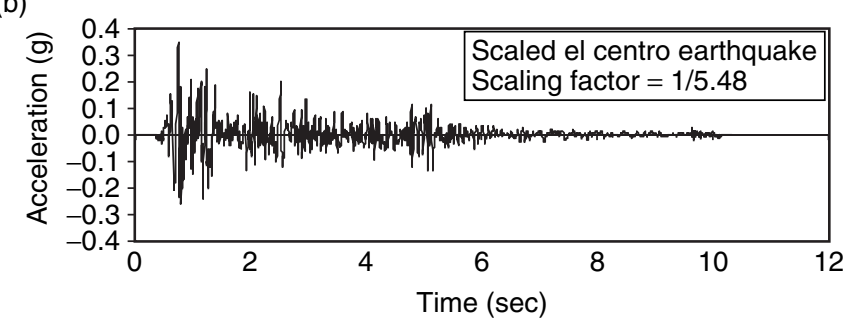

Figure 11. El Centro earthquake (1940): (a) original record; (b) scaled record

(a)

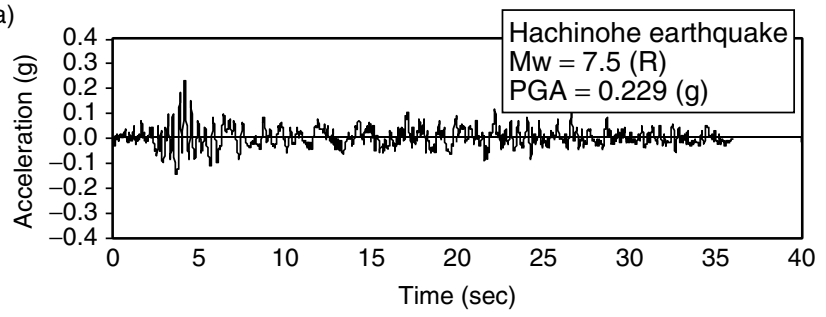

(b)

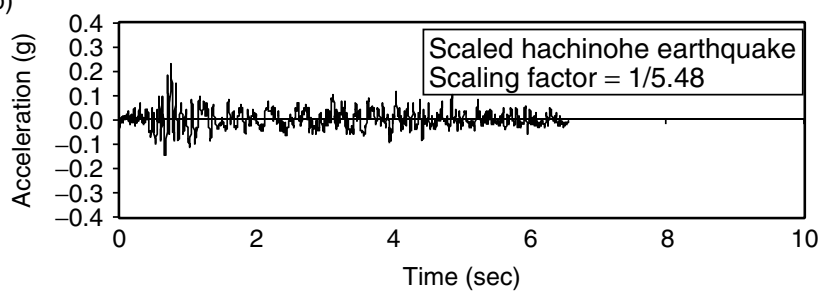

Figure 12. Hachinohe earthquake (1968): (a) original record; (b) scaled record

and natural frequency of the prototype $\left(f_{b}\right)$ is 5.48 while scaling relations between the model and prototype accelerations is 1.0 , meaning the earthquake magnitude remains the same as the prototype. Therefore, for scaling the earthquake records, it is required to reduce the time steps of the original records by factor of 5.48. As a result, the original time steps of Kobe, Northridge, and El Centro earthquake acceleration records were shifted from 0.02 to 0.00365 , while for Hachinohe earthquake record, the original time steps of 0.01 shifted to 0.001825 . The scaled acceleration records of the four adopted earthquakes are illustrated in Figures 9(b) to 12(b).

\section{SHAKING TABLE TESTS ON FIXED BASE STRUCTURAL MODEL}

Tests were carried out on the constructed structural model, described in Section 6.1, as a fixed base model (structure directly fixed on top of the shaking table) in order to ensure the structural model possesses the targeted natural frequency and determine the damping ratio of the structural model. In addition, to verify the numerical model seismic response of the fixed base model under the influence of the four scaled earthquake records were obtained. To achieve the above, constructed structural model was fixed and secured on the UTS shaking table. After securing the structural model on the shaking table, instrumentations including displacement transducers and accelerometers were installed on the structure in order to monitor the behaviour of the structure and to primarily measure structural lateral displacements. It should be noted that in addition to the displacement transducers installed at levels $3,5,7,11,13$, and 15 , eight accelerometers were installed at levels $3,5,7,9,11,13$, and 15 so as to check the consistency of the recorded displacements. Displacement, acceleration and velocity in time domain are closely related to each other. If the measured parameter is acceleration, displacement can be found through a double integration in time domain. Therefore, displacements of the various levels were determined by integrating the corresponding accelerations, measured by the accelerometers, in time domain and checked against the recorded displacements to ensure the consistency and accuracy of the obtained records. Figure 13 illustrates the final arrangement of the displacement transducers and accelerometers at different levels of the structural model.

Table 4. Utilised earthquake ground motions

\begin{tabular}{lcccccc}
\hline Earthquake & Country & Year & PGA (g) & Mw (R) & T (S) duration & Type \\
\hline Northridge & USA & 1994 & 0.843 & 6.7 & 30.0 & Near field \\
Kobe & Japan & 1995 & 0.833 & 6.8 & 56.0 & Near field \\
El Centro & USA & 1940 & 0.349 & 6.9 & 56.5 & Far field \\
Hachinohe & Japan & 1968 & 0.229 & 7.5 & 36.0 & Far field \\
\hline
\end{tabular}




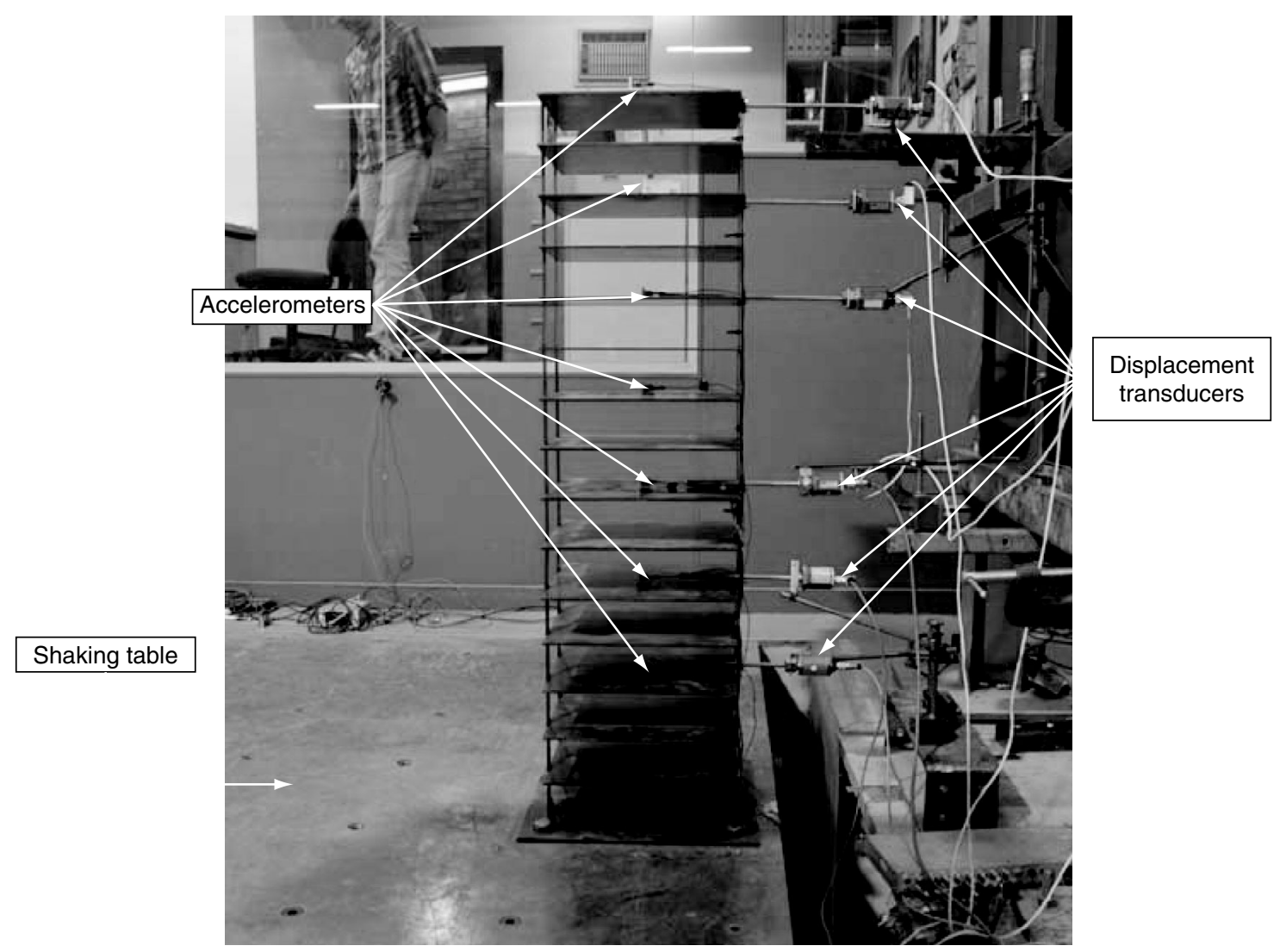

Figure 13. Final arrangement of the measuring instruments of the fixed base model

Initially, Sine Sweep test was performed on the structural model to determine the natural frequency of the model. Sine Sweep test involves a logarithmic frequency sweep holding a specified acceleration constant at the base of the structure. For the current Sin Sweep test, frequency of the shaking table has increased from $0.1 \mathrm{~Hz}$ to $50 \mathrm{~Hz}$. The first resonance between the shaking table and structural model frequencies showed the fundamental natural frequency of the model. The test was repeated three times to ensure the determined natural frequency is adequately accurate. The resulting natural frequency of the constructed structural model obtained from sin sweep test results was $2.19 \mathrm{~Hz}$ which is in a very good agreement with the desired natural frequency of the structural model (Table 2). Therefore, the constructed structural model, with the natural frequency $\left(f_{m}\right)$ of $2.19 \mathrm{~Hz}$ and the total mass $\left(m_{m}\right)$ of $104 \mathrm{~kg}$, possesses the required characteristics as summarised in Table 2, to meet the dynamic similarity criteria. The estimated value of the structural damping ratio of the constructed structural model has been determined from the free vibration lateral displacement records of the structural model using the following Taylor series expansion (Roy et al. 2006):

$$
\begin{aligned}
\frac{U_{n}}{U_{n+m}} & =e^{2 \pi m \xi}=1+\sum_{n=1}^{\infty} \frac{(2 \pi m \zeta)^{n}}{n !} \\
& =1+2 \pi m \xi+\frac{(2 \pi m \xi)^{2}}{2 !}+\ldots
\end{aligned}
$$

where, $\xi$ is the structural damping ratio and $U_{n}$ and $U_{n+m}$ are two positive peaks of the free vibration response of the structure which are $m$ cycles apart. Substituting the values of $U_{n}$ and $U_{n+m}$ for the two positive peaks of the free vibration lateral displacement records in Eqn 8, which are 10 cycles apart, and repeating the whole process several times, the estimated structural damping ratio $(\xi)$ is $1.1 \%$.

After ensuring adequacy of the structural model characteristics, shaking table tests were performed by applying scaled earthquake acceleration records of Kobe, 1995 [Figure 9(b)], Northridge, 1994 [Figure 10(b)], El Centro, 1940 [Figure 11(b)], and Hachinohe, 1968 [Figure 12(b)] to the fixed base structural model. The results of the performed shaking table tests under the influence of four scaled earthquake acceleration records in terms of maximum lateral deflections are 
determined and presented in Figure 16. In determination of the lateral deflections, the movement of the shaking table has been subtracted from storey movements. Therefore, all the records are in comparison to the base movements. It should be noted that for the sake of accuracy and consistency, the recorded displacements using displacement transducers, verified against the calculated displacements from accelerometer records, have been presented.

\section{SHAKING TABLE TESTS ON SOIL-STRUCTURE MODEL}

The first step in setting up the main phase of the shaking table tests, was securing the constructed laminar soil container on the UTS shaking table. For this purpose, the soil container was placed at the designated location, then fixed and secured on the shaking table using eight M38 bolts passing through the provided holes. The internal surface of the soil container then was covered and sealed with two layers of black plastic sheeting. Similar to Gohl and Finn (1987) and Valsangkar et al. (1991), $25 \mathrm{~mm}$ thick absorbing layers of Polystyrene foam sheets have been installed at the end walls of the soil container to simulate viscous boundaries in the free field condition. The thick layers of Polystyrene minimise reflection of outward propagating waves back into the model and allow the necessary energy radiation.
In addition, a layer of well graded gravelly soil particles were glued to the bottom of the soil container to simulate the frictional contact between the soil and the bedrock. This layer provides friction between the timber base plate (as the bedrock) and the in-situ soil mix and does not allow the soil mix to slip over the base plate. Various components of the laminar soil container are shown in Figure 14.

As explained in Section 6.3, the selected soil mix obtains the required stiffness and consequently the shear wave velocity after two days of curing. As a result, the time schedule of the testing process was highly intensive and time sensitive. Therefore, soil mixing and placement needed to be carried out in one day in order to produce a homogenous soil mix and after two days of curing, the final tests were to be performed.

$2 \mathrm{~m}^{3}$ of the soil mix (kaolinite, bentonite, fly ash, lime, and water) was produced and placed into the laminar soil container. During the soil mixing process, ten cylindrical soil samples of $D=50 \mathrm{~mm}$ and $h=100 \mathrm{~mm}$ were taken from the soil mix for quality control of the mix. The entire mixing process and filling the laminar soil container were completed in one day. Then, the soil mix inside the container was left to be cured for two days while the surface of the soil container was covered and sealed. On the second day, the structural model was lifted up and placed on the designated location, exactly

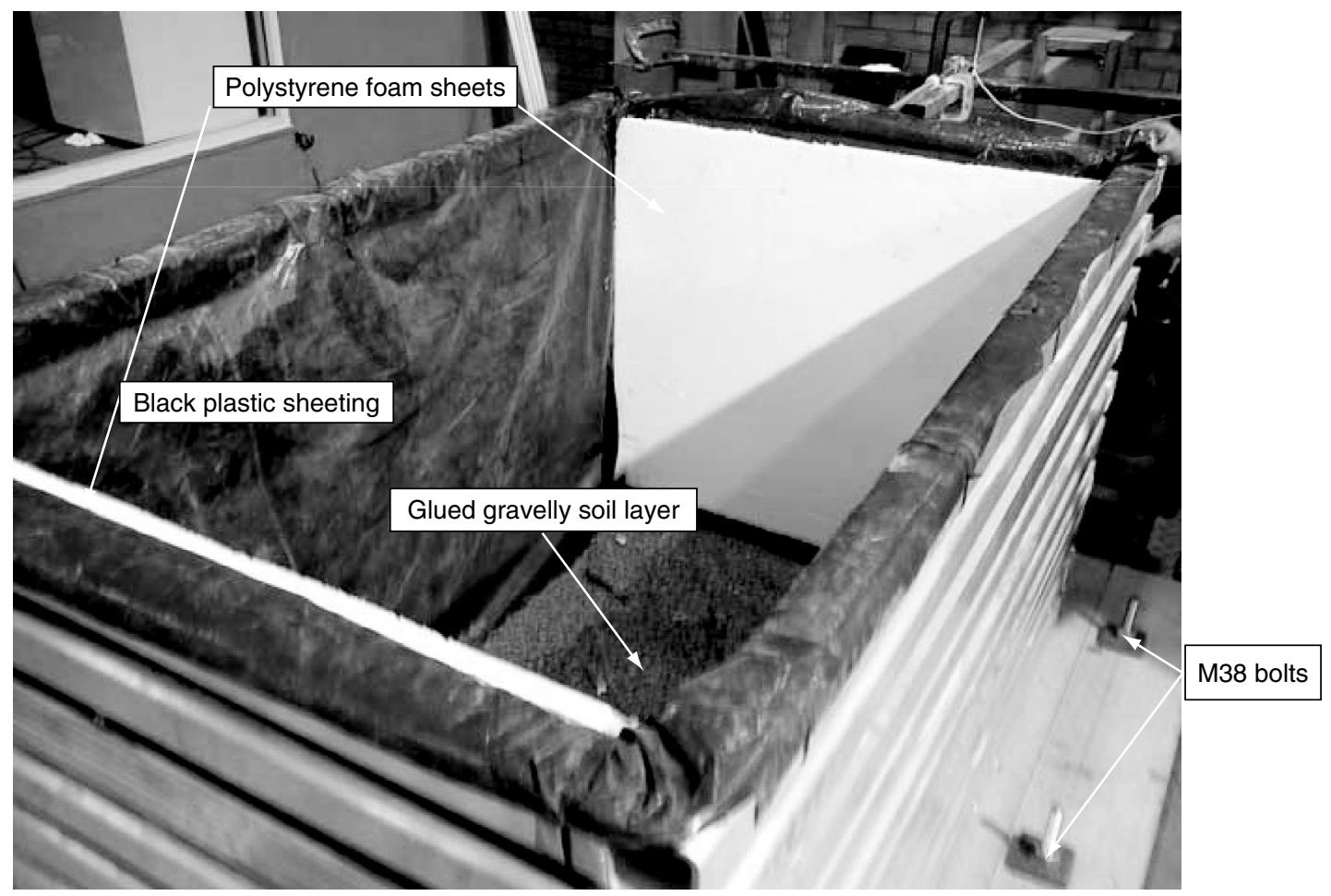

Figure 14. Various components of the secured laminar soil container on the shaking table 
in the middle of the soil surface. After securing the structural model on top of the soil, no excessive settlement or failure was observed underneath the base plate, indicating that the shear strength of the soil mix was adequate to carry the weight of the structural model, as expected and examined in Section 6.3. Instrumentation of the structure in the soil-structure system has been similar to the fixed base structure, as explained in Section 8 (Figure 13). In addition, vertical displacement transducers were placed on the level base plate of the structure (simulating the foundation) to determine the vertical displacements of the structure during the testing process. Figure 15 shows the final setup of the displacement transducers and accelerometers at different levels of the structural model for the soil-structure system on the shaking table.

In this study, in addition to lateral displacements, numerical fixed-base and flexible base frequencies were checked and verified against experimental measurements. Using the results of Sin Sweep test and comparing the shaking table frequencies with and without the structure model, pure fundamental fixed base frequencies have been extracted in this study. Sine Sweep test involves a logarithmic frequency sweep holding a specified acceleration constant at the base of the structure. For the current Sin Sweep test, frequency of the shaking table has increased from $0.1 \mathrm{~Hz}$ to $50 \mathrm{~Hz}$. The first resonance between the shaking table and structural model frequencies showed the fundamental natural frequency of the model. The test was repeated three times to ensure the determined natural frequency is adequately accurate. The obtained natural frequencies of the fixed base structure, and soil-structure model from the performed Sin Sweep tests were estimated to be $2.19 \mathrm{~Hz}$, and $1.60 \mathrm{~Hz}$, respectively. The corresponding values obtained from the numerical analysis, are 2.11 and 1.55 , respectively, which are in a good agreement with the laboratory measurements. As expected, natural frequency of the soil-structure model is considerably smaller than the natural frequency of the fixed base structural model. Afterwards, shaking table tests were undertaken by applying scaled earthquake acceleration records of Kobe, 1995 [Figure 9(b)], Northridge, 1994 [Figure 10(b)], El Centro, 1940 [Figure 11(b)], and Hachinohe, 1968 [Figure 12(b)] to the flexible base model, with the final setup as shown in Figure 15. The results of the carried out shaking table tests under the influence of four scaled earthquake acceleration records in terms of the maximum lateral deflections of various storey of the structure are illustrated in Figure 16. Figure 16 illustrates an example of experimental time-history displacement results for fixed base and flexible base

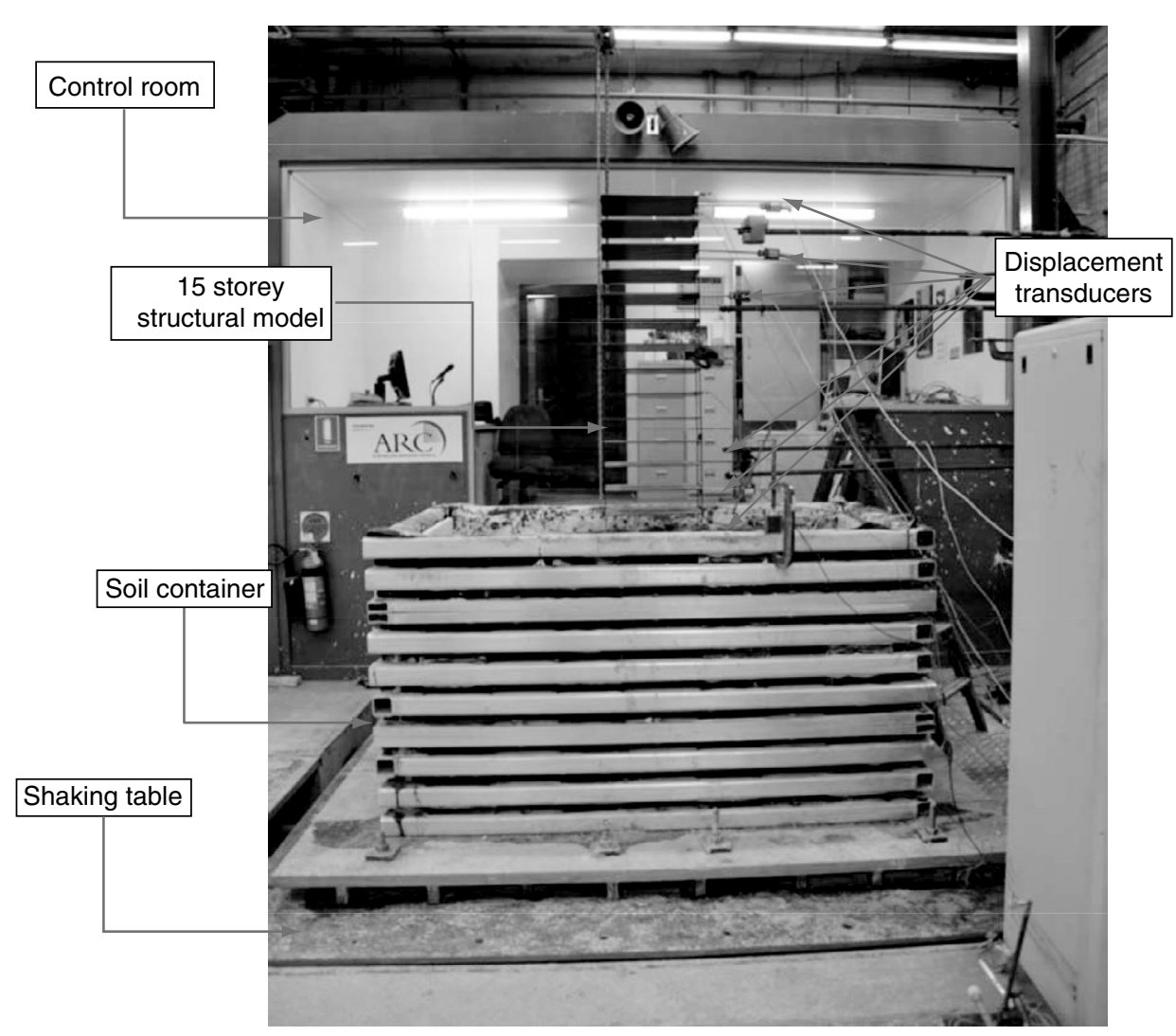

Figure 15. Final setup of the measuring instruments of the soil-structure model 


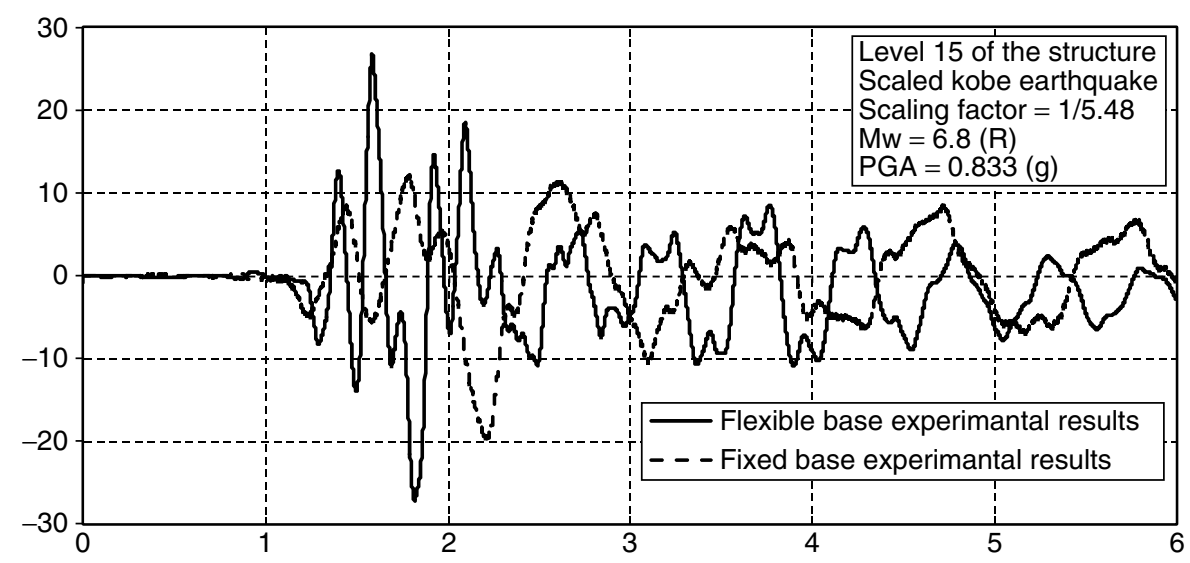

Figure 16. Sample experimental time-history displacement results in millimetres for fixed base and flexible base models under the influence of Kobe earthquake (1995) at level 15

models under the influence of Kobe earthquake (1995). In addition, the maximum vertical displacements of the base plate have been obtained from the vertical displacement transducers installed at the level of the base plate for each earthquake record, respectively, and summarised in Table 7.

\section{NUMERICAL MODELLING OF SHAKING TABLE TESTS}

Numerical modelling of the fixed base and flexible base (soil-structure) models have been carried out in two stages. The numerical model of the constructed structural model, shown in Figure 6, was built in FLAC2D using dimensions of the physical model. After building the geometry of the structural model, the required structural parameters including crosssectional area of the beams $\left(A_{b}\right)$, moment of inertia of the beams $\left(I_{b}\right)$, cross-sectional area of the columns $\left(A_{c}\right)$, moment of inertia of the columns $\left(I_{c}\right)$, crosssectional area of the foundation slab $\left(A_{s}\right)$, moment of inertia of the foundation slab $\left(I_{s}\right)$, modulus of elasticity of steel $(E)$, density $(\rho)$, and structural damping ratio $(\xi)$, summarised in Table 5 , were extracted from the construction detail drawings and specifications and adopted in the numerical simulation of the structure in FLAC2D. Figure 17(a) illustrates the created model that numerically defines the geometry, properties, and loading of the physical fixed base model in FLAC2D.
In order to simulate flexible base (soil-structure) model in FLAC2D, the proposed soil-structure model, explained in Section 2, has been employed. The summarised structural characteristics in Table 5 have been adopted to simulate the structural part of the flexible base model. As reported in Section 9, ten cylindrical soil specimens of size $D=50 \mathrm{~mm}$ and $h=$ $100 \mathrm{~mm}$ were successively taken from the soil mix, during the soil mixing process. In order to adopt the most accurate soil parameters in simulation of the physical soil-structure model, shear wave velocity $\left(V_{s}\right)$ and soil density $(\rho)$ of the samples in the second day of curing were determined by performing bender element and density tests on the UTS soils laboratory. The average results of the ten specimens indicated that the values of shear wave velocity $\left(V_{s}\right)$ and soil density $(\rho)$ were $35.5 \mathrm{~m} / \mathrm{s}$ and $1450 \mathrm{~kg} / \mathrm{m}^{3}$, respectively. These results have been in very good agreement and conformity to the initial laboratory test results, summarised in Table 3. The adopted soil properties in the numerical simulation of the flexible base model consist of shear strength $\left(S_{u}\right)$, shear wave velocity $\left(V_{s}\right)$, low strain shear modulus $\left(G_{\max }\right)$, bulk modulus $(K)$, and density $(\rho)$, summarised in Table 6 .

After creating fixed base and flexible base numerical models in FLAC2D (Figure 17), fully nonlinear time history dynamic analyses were carried out on both fixed base and flexible base models under the influence of four scaled earthquake acceleration records including

Table 5. Adopted parameters for numerical simulation of the structural model

\begin{tabular}{lcccccccc}
\hline $\begin{array}{l}\boldsymbol{A}_{\boldsymbol{b}} \\
\left(\mathbf{m}^{2}\right)\end{array}$ & $\begin{array}{c}\boldsymbol{I}_{\boldsymbol{b}} \\
\left(\mathbf{m}^{4}\right)\end{array}$ & $\begin{array}{c}\boldsymbol{A}_{\boldsymbol{c}} \\
\left(\mathbf{m}^{2}\right)\end{array}$ & $\begin{array}{c}\boldsymbol{I}_{\boldsymbol{c}} \\
\left(\mathbf{m}^{\mathbf{4}}\right)\end{array}$ & $\begin{array}{c}\boldsymbol{A}_{\boldsymbol{s}} \\
\left(\mathbf{m}^{2}\right)\end{array}$ & $\begin{array}{c}\boldsymbol{I}_{\mathbf{s}} \\
\left(\mathbf{m}^{4}\right)\end{array}$ & $\begin{array}{c}\boldsymbol{E} \\
(\mathbf{k P a})\end{array}$ & $\begin{array}{c}\rho \\
\left(\mathbf{k g} / \mathbf{m}^{3}\right)\end{array}$ & $\xi(\%)$ \\
\hline 0.002 & $4.16 \mathrm{E}-9$ & $1.6 \mathrm{E}-4$ & $5.33 \mathrm{E}-11$ & 0.005 & $4.16 \mathrm{E}-8$ & $2.0 \mathrm{E} 8$ & 7850 & 1.1 \\
\hline
\end{tabular}


(a)

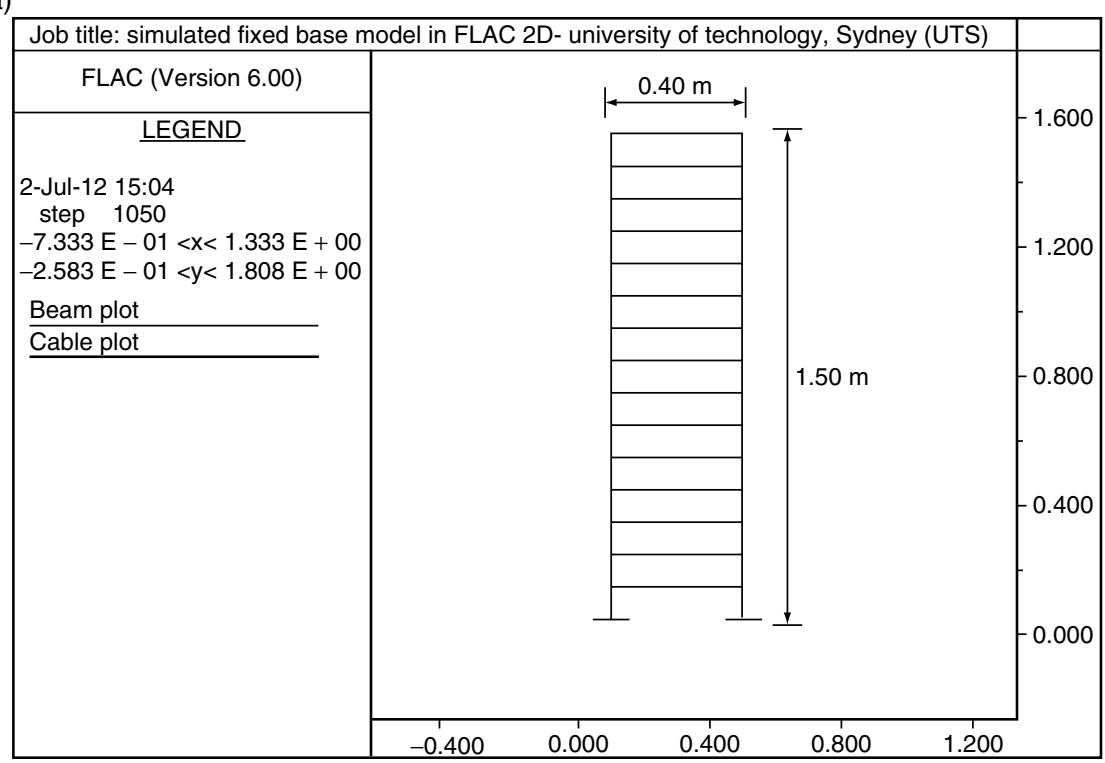

(b)

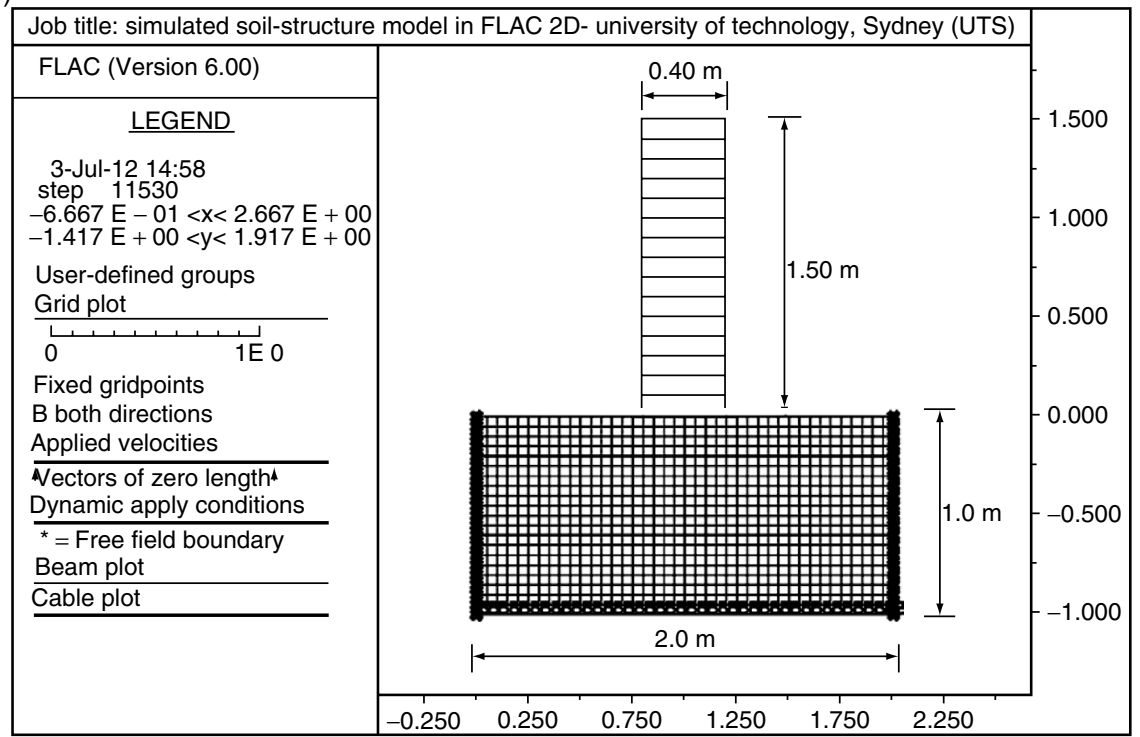

Figure 17. Simulated numerical model in FLAC2D: (a) fixed base model; (b) flexible base model

Table 6. Adopted soil parameters in numerical simulation of soil-structure model

\begin{tabular}{lccccc}
\hline Parameters & $\begin{array}{c}\boldsymbol{S}_{\boldsymbol{u}} \\
(\mathbf{k P a})\end{array}$ & $\begin{array}{c}\boldsymbol{V}_{\boldsymbol{s}} \\
(\mathbf{m} / \mathbf{s})\end{array}$ & $\begin{array}{c}\boldsymbol{G}_{\mathrm{max}} \\
(\mathbf{k P a})\end{array}$ & $\begin{array}{c}\boldsymbol{K} \\
(\mathbf{k P a})\end{array}$ & $\begin{array}{c}\rho \\
\left(\mathbf{k g} / \mathbf{m}^{\mathbf{3}}\right)\end{array}$ \\
\hline Values & 1.57 & 35.5 & 1830 & 90760 & 1450 \\
\hline
\end{tabular}

Kobe, 1995 [Figure 9(b)], Northridge, 1994 [Figure 10(b)], El-Centro, 1940 [Figure 11(b)], and Hachinohe, 1968 [Figure 12(b)].

Inelastic structural analysis was performed by introducing the plastic moments $\left(M^{P}\right)$ for the structural sections. The values of the plastic moments have been calculated by considering a flexural structural member of width $b$ and height $h$ with yield stress $\sigma_{y}$ using Eqn 9 as follows:

$$
M^{P}=\sigma_{y}\left(\frac{b h^{2}}{4}\right)
$$

In the inelastic structural analysis it is assumed that structural elements behave elastically until reaching the defined plastic moment. The section at which the plastic moment $\left(M^{P}\right)$ is reached can continue to deform, without inducing additional resistance. In addition, geometric nonlinearity of the structures, capturing 
P-Delta effects, has been accommodated by specifying large-strain solution mode in FLAC2D software in the structural analyses of fixed base and flexible base models.

In the soil-structure model, the built-in tangent modulus function presented by Hardin and Drnevich (1972), known as Hardin model is employed in order to implement hysteretic damping to the model. Adopted model in FLAC2D generates backbone curves representing Sun et al. (1998) curves for clay, adopting $\gamma_{r e f}=0.234$ (Figure 18) as the numerical fitting parameter. In this way, nonlinear behaviour of the subsoil has been considered in the dynamic analysis. Afterwards, the numerical results of the inelastic time history dynamic analyses under the influence of the four mentioned scaled earthquake acceleration records in terms of the maximum inelastic lateral deflections and the maximum inelastic vertical displacements of the base plate were determined for both fixed base and flexible base models from FLAC2D displacement history records for each scaled earthquake. Then, results of the numerical fixed base and flexible base models were compared with the experimental results of the shaking table tests performed on the fixed base model and the flexible base model. The mentioned results are shown in Figure 19 for fixed based and flexible base models.

\section{RESULTS AND DISCUSSIONS}

The numerical predictions and experimental values of the maximum lateral displacements of the fixed base and the flexible base models are presented and compared in Figure 19. Average values of the numerical predictions and experimental values of the maximum lateral displacements of the fixed base and the flexible base models were determined and compared in Figure 20(a), while their corresponding inter-storey drifts have been calculated using the following equation based on AS 1170.4-2007 (Earthquake Actions in Australia):

(a)

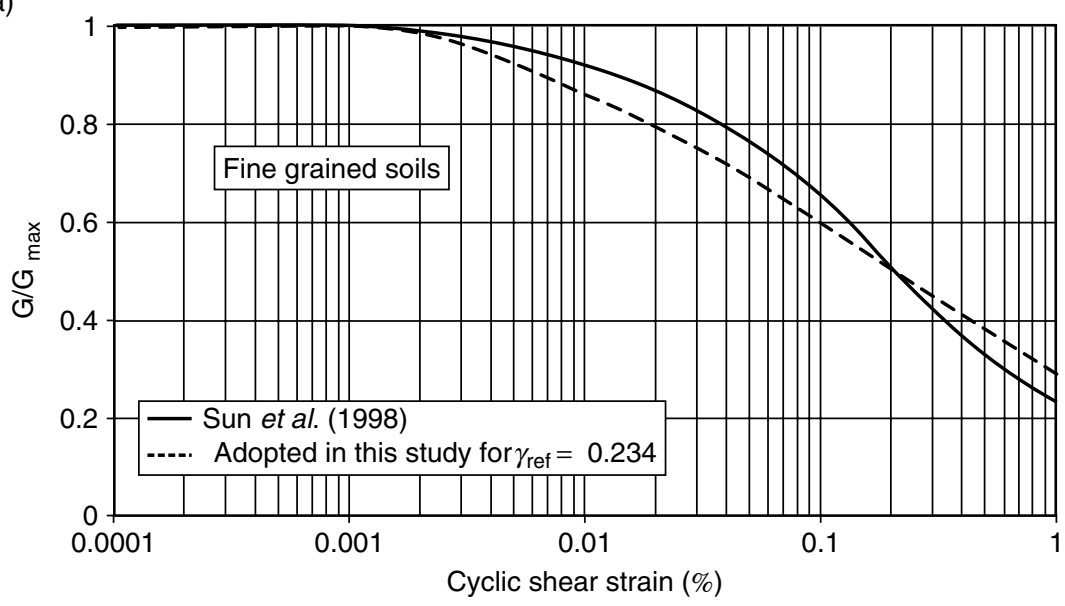

(b)

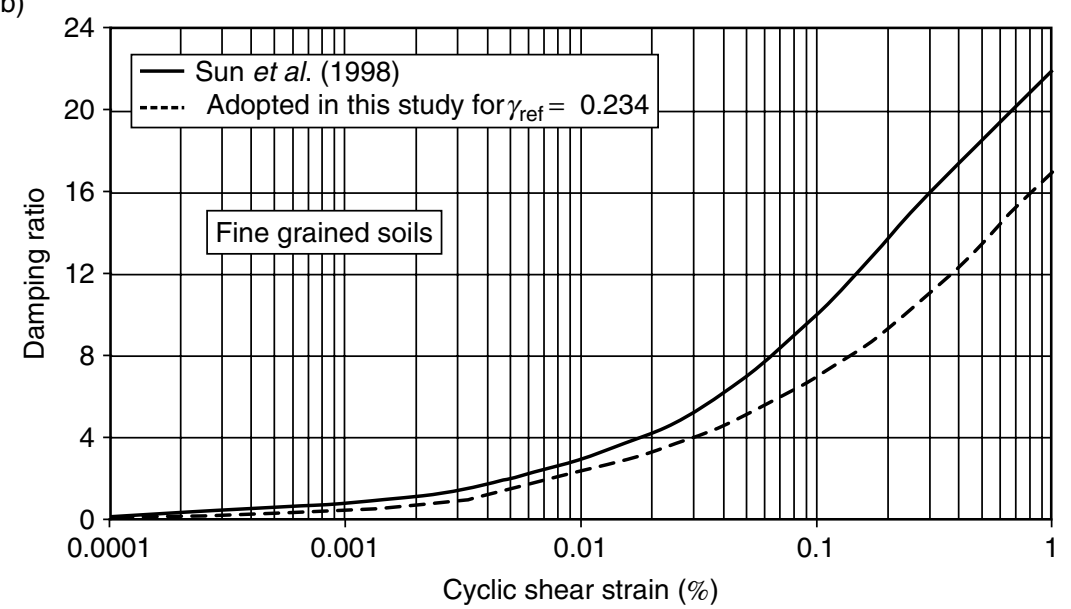

Figure 18. Adopted fitting curves for clay in this study: (a) relations between $G / G_{\max }$ versus cyclic shear strain; (b) relations between material damping ratio versus cyclic shear strain 
(a)

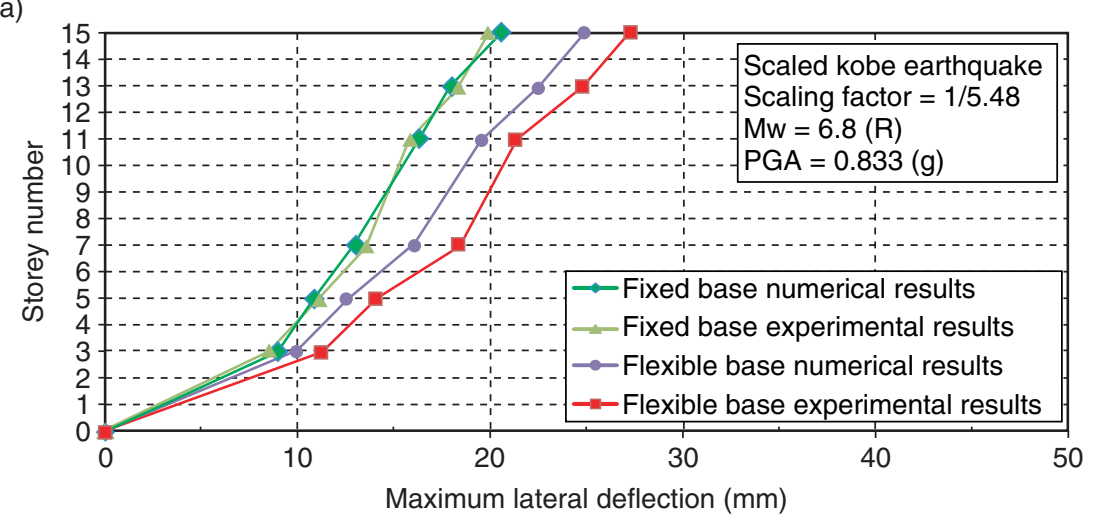

(b)

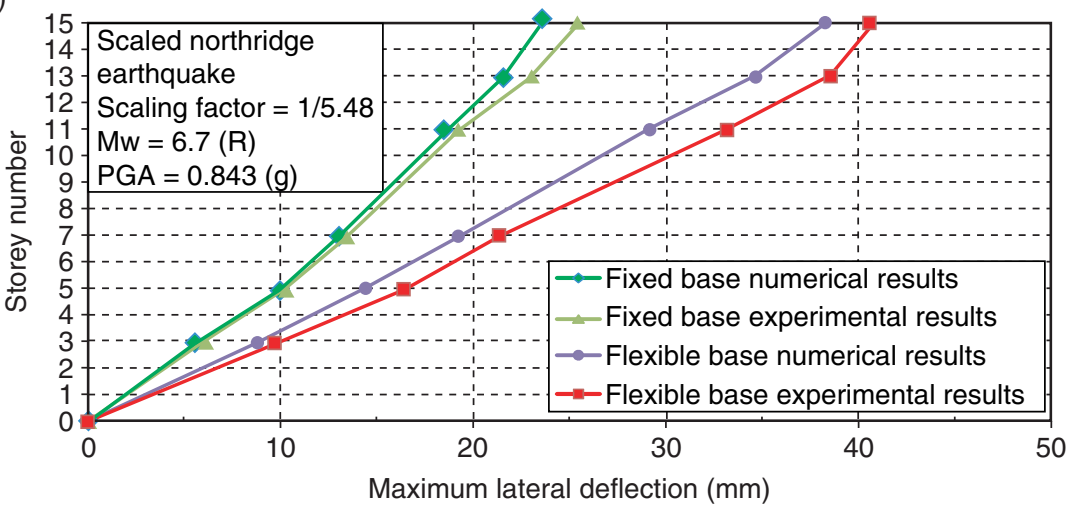

(c)

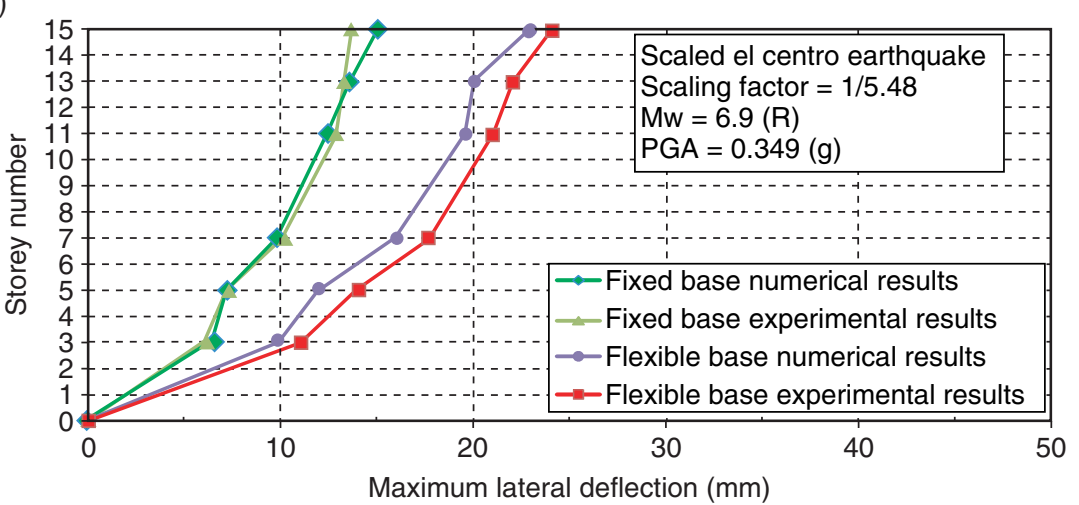

(d)

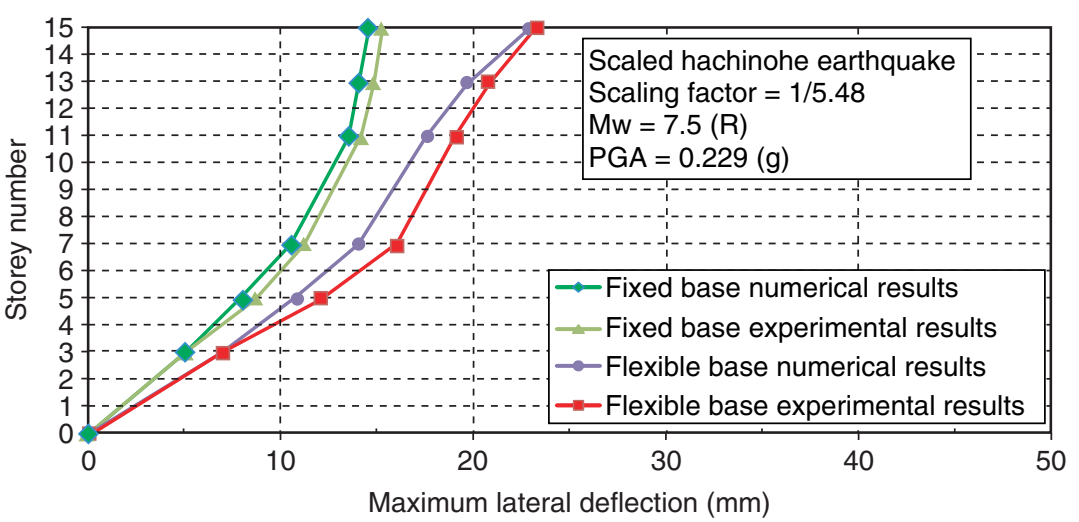

Figure 19. Numerical and experimental maximum lateral displacements of fixed base and flexible base models under the influence four different scaled earthquake records: (a) Kobe (1995) earthquake; (b) Northridge (1994) earthquake; (c) El Centro (1940) earthquake;

(d) Hachinohe (1968) earthquake 
(a)

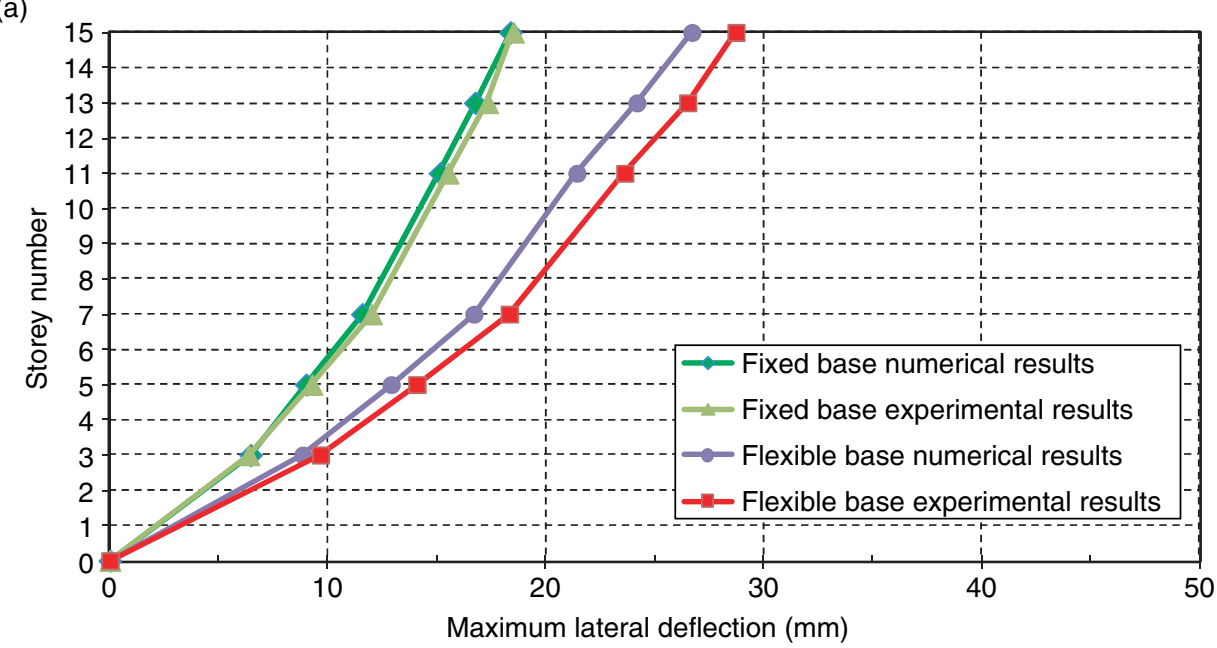

(b)

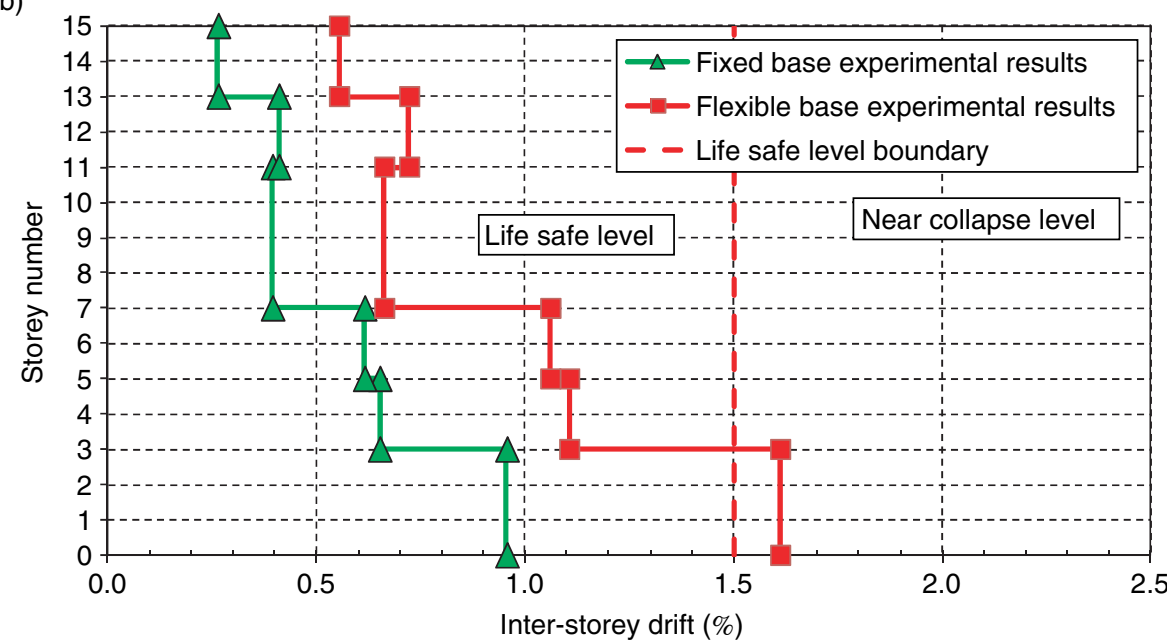

Figure 20. (a) Average values of the numerical predictions and experimental values of the maximum lateral displacements of fixed base and flexible base models; (b) Average experimental inter-storey drifts of fixed base and flexible base models

$$
\text { drift }=\left(d_{i+1}-d_{i}\right) / h
$$

where, $d_{i+1}$ is deflection at $(i+1)$ level, $d_{i}$ is deflection at (i) level, and $h$ is the storey height.

The average values of numerical and experimental inter-storey drifts, determined by Eqn 10, are illustrated in Figure 20(b). In addition, the predicted and measured vertical displacements of the base plate are summarised and compared in Table 7.

Comparing the predicted and observed values of the maximum lateral displacements of the fixed base and the flexible base models under the influence of the four mentioned scaled earthquake acceleration records (Figure 19), the accuracy of the numerical fixed base and flexible base model is examined. Accordingly, it becomes apparent that the trend and the values of the numerical seismic response, predicted by the fixed base numerical model as well as the new developed numerical soil-structure model, are in a good agreement and consistent with the experimental shaking table test results.

Based on the experimental average values of maximum lateral deflections of the fixed base and the flexible base models [Figure 20(a)], lateral deflections of flexible base models have increased by $55 \%$ in comparison to fixed base model. According to Kramer (1996), relative lateral structural displacements under the influence of soil-structure interaction consist of rocking component and distortion component. Any change in the displacements is an outcome of changes in these components. In this particular case, considering the maximum foundation rotation values summarised in Table 7 and maximum lateral displacements reported in Figure 20(a), it is noted that approximately $55 \%$ of the maximum lateral deflections of the flexible base model [Figure 20(a)] were due to the rocking component, 
Table 7. Numerical and experimental maximum vertical displacements and rotations

\begin{tabular}{lcccc}
\hline & \multicolumn{2}{c}{ Maximum vertical displacement } & \multicolumn{2}{c}{ Maximum foundation rotation } \\
\hline $\begin{array}{l}\text { Scaled } \\
\text { earthquake }\end{array}$ & $\begin{array}{l}\text { Numerical } \\
\text { prediction }\end{array}$ & $\begin{array}{l}\text { Experimental } \\
\text { measurement }\end{array}$ & $\begin{array}{l}\text { Numerical } \\
\text { prediction }\end{array}$ & $\begin{array}{l}\text { Experimental } \\
\text { measurement }\end{array}$ \\
\hline Kobe & $2.33 \mathrm{~mm}$ & $2.54 \mathrm{~mm}$ & $0.54^{\circ}$ & $0.58^{\circ}$ \\
Northridge & $1.22 \mathrm{~mm}$ & $1.32 \mathrm{~mm}$ & $0.28^{\circ}$ & $0.30^{\circ}$ \\
El-Centro & $1.85 \mathrm{~mm}$ & $1.98 \mathrm{~mm}$ & $0.42^{\circ}$ & $0.45^{\circ}$ \\
Hachinohe & $1.40 \mathrm{~mm}$ & $1.47 \mathrm{~mm}$ & $0.32^{\circ}$ & $0.33^{\circ}$ \\
\hline
\end{tabular}

while $45 \%$ took place due to the distortion component. For example, under the influence of Northridge (1994) earthquake, maximum lateral deflection at the top of the fixed base model was measured to be $25.3 \mathrm{~mm}$ due to distortion component, while maximum lateral deflection at the top of the flexible base model was $40.6 \mathrm{~mm}$ which $22.5 \mathrm{~mm}$ of that value was due to rocking component and $18.1 \mathrm{~mm}$ took place due to distortion component. It should be noted that in order to determine rocking and distortion components, Trifunace et al. (2001) relationships have been used in this study. In other words, the rotation angle of the foundation has been used to predict the lateral displacement due to the rocking. Evidently, the maximum lateral distortion of the structure under the influence of soil-structure interaction decreases due to reduction in distortion component of the lateral displacement while the overall maximum lateral deflection increases as the rocking component is included. It can be concluded that, soilstructure interaction increases the overall lateral displacements while reduces the lateral distortions of moment resisting building frames resting on relatively soft soils.

As shown in Figure 20(b), due to amplification of the experimental average values of maximum lateral deflections due to SSI [Figure 20(a)], performance level of the structural model changes significantly from life safe to near collapse level. Such a considerable change in the performance level of the model is extremely dangerous and safety threatening. Thus, in the examined experimental investigation, dynamic soil-structure interaction has profound effects on the seismic response of the structural model resting on relatively soft soil.

Reviewing the average maximum lateral deflections [Figure 20(a)] and maximum vertical displacements and rotations (Table 7), it becomes apparent that the numerical predictions and laboratory measurements are in a good agreement (less than $10 \%$ difference). Therefore, the numerical soil-structure model can replicate the behaviour of the real soil-structure system with acceptable accuracy. The observed discrepancy between the numerical predictions and laboratory observations could be due to the variation of soil properties such as shear wave velocity and shear modulus with depth occurring during mixing and placement process. In addition, energy absorption at the bolted connection of the base in the physical laboratory model which cannot be captured by rigid base assumption of the numerical model may be another reason for the observed discrepancy.

It should be noted that increasing the overall drift caused by rocking component increases $\mathrm{P}-\Delta$ effect. $\mathrm{P}-\Delta$ effect is a destabilising moment equal to the force of gravity multiplied by the horizontal displacement a structure undergoes as a result of a lateral displacement. To illustrate the effect, take the example of a typical statics case: in a perfectly rigid body subjected only to small displacements, the effect of a gravitational or concentrated vertical load at the top of the structure is usually neglected in the computation of ground reactions. However, structures in real life are flexible and can exhibit large lateral displacements. Given the side displacement, the vertical loads present in the structure can adversely perturb the ground reactions. As a result of the overall lateral displacement amplifications and consequent $\mathrm{P}-\Delta$ effect, it is observed in this study that the performance level of the structure changes from life safe to near collapse level which is very dangerous and safety threatening. In addition, increasing the overall drifts will have destructive effects on non-structural components of the system which should be seen and addressed by a safe structural design.

\section{CONCLUSIONS}

Based on the experimental and numerical investigations conducted in this study, the predicted results from the proposed numerical models were in a good agreement with the laboratory measurements. Thus, the numerical soilstructure model can replicate the behaviour of the real soilstructure system with acceptable accuracy. It is concluded that the proposed numerical soil-structure model is a valid and qualified method of simulation with sufficient accuracy which can be employed for further numerical dynamic soil-structure interaction investigations. 
In addition, it is understood that the predicted and measured lateral deflections of the flexible base model have noticeably amplified in comparison to the fixed base model. Approximately 55\% of the lateral deformations were due to the rocking component, while $45 \%$ took place due to the distortion component. Therefore, soil-structure interaction increases the overall lateral displacements while reduces the lateral distortions of moment resisting building frames resting on relatively soft soils. As a result of the overall lateral deflection amplifications, it is observed that the performance level of the structural model may change from life safe to near collapse level which is very dangerous and safety threatening. Thus, soil-structure interaction has considerable effects on the seismic response of moment resisting building frames resting on relatively soft soils and should be take into consideration in the seismic design.

In this study, it is observed that base shear of the structures modelled with soil as flexible base are generally less than the base shear of the structures modelled as fixed base. Base shear decreases due to reduction in lateral distortions. However, as a consequence of overall lateral deflections, the corresponding inter-storey drifts of flexible base models increase profoundly.

It can be concluded that the conventional design procedure excluding SSI may not be adequate to guarantee the structural safety of mid-rise moment resisting building frames resting on relatively soft soil deposits. As most of the seismic design codes around the globe do not address the soil-structure interaction (SSI) explicitly, considering SSI effects in the seismic designs as a distinguished part of these standards is highly recommended. It is also recommended to engineering companies working in regions located in high earthquake risk zones, to consider dynamic soilstructure interaction effects in the analysis and design of mid-rise moment resisting building frames resting on soft soils to ensure safety of the design.

\section{REFERENCES}

AS1170.4-2007 (2007). Structural Design Actions - Part 4:

Earthquake Actions in Australia, Australian Standards, Sydney.

AS 1289.3.5.1-2006 (2006). Methods of Testing Soils for Engineering

Purposes, Determination of the Soil Particle Density of a Soil-

Standard Method, Australian Standards, Sydney.

AS5101.4-2008 (2008). Methods for Preparation and Testing of

Stabilised Materials, Method 4: Unconfined Compressive

Strength of Compacted Materials, Australian Standards, Sydney. AS/NZS 3678-2011 (2011). Structural Steel - Hot-Rolled Plates,

Floor Plates and Slabs, Australian Standards, Sydney.
Ashour, M., Norris, G. and Pilling, P. (1998). "Lateral loading of a pile in layered soil using the strain wedge model", Journal of Geotechnical and Geoenvironmental Engineering, ASCE, Vol. 124, No. 4, pp. 303-315.

Bi, K. and Hao, H. (2012). "Modelling and simulation of spatially varying earthquake ground motions at sites with varying conditions", Probabilistic Engineering Mechanics, Vol. 29, pp. 92-104.

Beaty, M.H. and Byrne, P.M. (2001). "Observations on the San Fernando Dams", Proceedings of the 4th International Conference on Recent Advances in Geotechnical Earthquake Engineering and Soil Dynamics, San Diego, California, March.

Byrne, P.M., Naesgaard, E. and Seid-Karbasi, M. (2006). "Analysis and design of earth structures to resist seismic soil liquefaction, in sea to sky geotechnique", Proceedings of the 59th Canadian Geotechnical Conference \& 7th Joint CGS/IAH-CNC Groundwater Specialty Conference, Vancouver, Canada, October, pp. 1-24.

Chau, K.T., Shen, C.Y. and Gou, X. (2009). "Nonlinear seismic soilpile-structure interactions: shaking table tests and FEM analyses", Soil Dynamics and Earthquake Engineering, Vol. 29, No. 2, pp. 300-310.

Cheung, W.M., Qin, X., Chouw, N., Larkin, T. and Orense, R. (2013). "Experimental and numerical study of soil response in a laminar box", Proceeding of 2013 NZSEE Conference, Wellington, New Zealand, April.

Chu, D. (2006). Three-Dimentional Nonlinear Dynamic Analysis of Soil-Pile-Structure Interaction, $\mathrm{PhD}$ Thesis, Washington University, Saint Louis, Missouri, USA.

Galal, K. and Naimi, M. (2008). "Effect of conditions on the response of reinforced concrete tall structures to near fault earthquakes", The Structural Design of Tall and Special Buildings, Vol. 17, No. 5, pp. 541-562.

Gazetas, G. and Mylonakis, G. (1998). "Seismic soil-structure interaction: new evidence and emerging issues", Geotechnical Special Publication 75, Geotechnical Earthquake Engineering and Soil Dynamics III, American Society of Civil Engineers, Reston, Virginia, pp. 1119-1174.

Gohl, W.B. and Finn, W.D.L. (1987). "Seismic response of single piles in shaking table studies", Proceedings of the Fifth Canadian Conference Earthquake Engineering, Rotterdam, pp. 435-444.

Hardin, B.O. and Drnevich, V.P. (1972). "Shear modulus and damping in soils: design equations and curves", Journal of Soil Mechanics and Foundation, Vol. 7, No. 8, pp. 667-692.

Ishimura, K., Ohtsuki, A., Yoloyama, K. and Koyanagi, Y. (1992). "Sway-rocking model for simulating nonlinear response of sandy deposit with structure", Proceedings of the Tenth World Conference on Earthquake Engineering, Madrid, Spain, pp. 1897-1903.

Itasca Consulting Group, Inc. (2008). FLAC2D: Fast Lagrangian Analysis of Continua User's Manual, Version 6.0, Minneapolis, USA.

Karamodin, A.K. and Kazemi, H.H. (2008). "Semi-active control of structures using neuro-predictive algorithm for mr dampers", 
Structural Control and Health Monitoring, Vol. 17, No. 3, pp. 237-253.

Kramer, S.L. (1996). Geotechnical Earthquake Engineering, Prentice Hall.

Krawinkler, H., Medina, R. and Alavi, B. (2003). "Seismic drift and ductility demands and their dependence on ground motions", Engineering Structures, Vol. 25, No. 5, pp. 637-653.

Langhaar, H. (1951). Dimensional Analysis and Theory of Models, John Wiley \& Sons Inc., New York, USA

Lee, C.H., Wei, Y. and Huo, Y. (2012). "Boundary effects of a laminar container in centrifuge shaking table tests", Soil Dynamics and Earthquake Engineering, Vol. 34, No. 1, pp. 37-51.

Lysmer, J. and Kuhlemeyer, R.L. (1969). "Finite dynamic model for infinite media", Journal of Engineering Mechanics, ASCE, Vol. 95, No. 6, pp. 859-877.

Meymand, P.J. (1998). Shaking Table Scale Model Tests of Nonlinear Soil-Pile-Superstructure Interaction in Soft Clay, $\mathrm{PhD}$ Thesis, Department of Civil Engineering, University of California, Berkeley, California, USA.

Moss, R.E.S., Crosariol, V. and Kuo, S. (2010). "Shake table testing to quantify seismic soil-structure interaction of underground structures", Proceedings of the 5th International Conference on Recent Advances in Geotechnical Earthquake Engineering and Soil Dynamics, San Diego, California, USA, May.

Prasad, S., Towhata, I., Chandradhara, G. and Nanjundaswamy, P. (2004). "Shaking table tests in earthquake geotechnical engineering", Current Science, Vol. 87, No. 10, pp. 1398-1404.

Pitilakis, D., Dietz, M., Wood, D.M., Clouteau, D. and Modaressi, A. (2008). "Numerical simulation of dynamic soil-structure interaction in shaking table testing", Soil Dynamics and Earthquake Engineering, Vol. 28, No. 6, pp. 453-467.

Pitilakis, K., Anastasiadis, A., Pitilakis, A. and Rovithis, E. (2013). "Full-scale testing of a model structure in Euroseistest to study soil-structure interaction", Proceedings of the 4th ECCOMAS Thematic Conference on Computational Methods in Structural Dynamics and Earthquake Engineering, Greece.

Qin, X., Cheung, W.M., Chouw, N., Larkin, T. and Ching, S.W.C. (2013). "Study of soil-structure interaction effect on ground movement using a laminar box", Proceeding of 2013 NZSEE Conference, Wellington, New Zealand, April.

Rayhani, M.H. and El Naggar, M.H. (2008). "Numerical modelling of seismic response of rigid foundation on soft soil", International Journal of Geomechanics, Vol. 8, No. 6, pp. 336-346.

Richards, J.R., Elms, D.G. and Budhu, M. (1990). "Dynamic fluidization of soils", Journal of Geotechnical Engineering, ASCE, Vol. 116, No. 5, pp. 740-759.

Roy, R., Craige, J.R. and Kurdila, A.J. (2006). Fundamentals of Structural Dynamics, $2^{\text {nd }}$ Edition, John Wiley \& Sons Inc, New Jersey.

Seo, C.G., Yuna, C.B. and Kimb, J.M. (2007). "Three-dimensional frequency-dependent infinite elements for soil-structure interaction", Engineering Structures, Vol. 29, No. 11, pp. 3106-3120.
Stanton, J.F., Banerjee, S. and Hasayen, I. (1998). Shaking Table Tests on Piles, Final report, Research Project Y-2811, Task 26, Prepared for Washington State Transportation Communication.

Sulaeman, A. (2010). The Use of Lightweight Concrete Piles for Deep Foundation on Soft Soils, PhD Thesis, Department of Civil Engineering, University of Tun Hussein Onn, Malaysia.

Sun, J.I., Golesorkhi, R. and Seed, B. (1998). Dynamic Module and Damping Ratios for Cohesive Soils, Earthquake Engineering Research Centre, Report No. UCB/EERC-88/15, University of California, Berkeley, California, USA.

Tabatabaiefar, H.R., Fatahi, B. and Samali, B. (2012). “An empirical relationship to determine lateral seismic response of mid-rise building frames under influence of soil-structure interaction", The Structural Design of Tall and Special Buildings. (in press)

Tabatabaiefar, H.R., Fatahi, B. and Samali, B. (2013). "Lateral seismic response of building frames considering dynamic soilstructure interaction effects", Structural Engineering and Mechanics, Vol. 45, No. 3, pp. 277-309.

Tang, L., Ling, X., Xu, P., Gao, X. and Wang, D. (2009). "Shake table test of soil-pile groups-bridge structure interaction in liquefiable ground", Earthquake Engineering and Engineering Vibration, Vol. 9, No. 1, pp. 1-12.

Taylor, C.A. (1997). Large Scale Shaking Tests of Geotechnical Structures, Earthquake Engineering Research Centre, University of Bristol.

Taylor, C.A., Dar, A.R. and Crewe, A.J. (1995). "Shaking table modelling of seismic geotechnical problems", Proceedings of the 10th European Conference on Earthquake Engineers, Vienna, Austria, pp. 441-446.

Trifunace, M. (1990). "How to model amplification of strong earthquake motions by local soil and geologic site conditions", Earthquake Engineering and Structural Dynamics, Vol. 19, No. 6, pp. 833-846.

Trifunace, M., Todorovska, M. and Hao, T. (2001a). "Full-scale experimental studies of soil-structure interaction - a review", Proceedings of the 2nd U.S.-Japan Workshop on Soil-Structure Interaction, Tsukuba City, Japan, March, pp. 1-52

Trifunac, M.D., Ivanović, S.S. and Todorovska, I. (2001b). “Appar ilding, II: Time-frequancy analysis", Journal of Structural Engineering, ASCE, Vol. 127, No. 5, pp. 527-537.

Turan, A., Hinchberger, S. and El Naggar, H. (2009). "Design and commissioning of a laminar soil container for use on small shaking tables", Soil Dynamics and Earthquake Engineering, Vol. 29, No. 2, pp. 404-414.

Turan, A., Hinchberger, S. and El Naggar, H. (2013). "Seismic soil-structure interaction in buildings on stiff clay with embedded basement stories", Canadian Geotechnical Journal, Vol. 50, No. 3, pp. 858-873.

Valsangkar, A.J., Dawe, J.L. and Mita, K.A. (1991). "Shake table studies of seismic response of single partially supported piles", Proceeding of the 6th Canadian Conference Earthquake Engineering, Toronto, Canada, June, pp. 327-334.

Wolf, J.P. (1985). Dynamic Soil-Structure Interaction, Prentice Hall Co, Englewood Cliffs, New Jersey, USA. 